\title{
THE DENSITY MANIFOLD AND CONFIGURATION SPACE QUANTIZATION
}

\author{
JOHN D. LAFFERTY
}

\begin{abstract}
The differential geometric structure of a Fréchet manifold of densities is developed, providing a geometrical framework for quantization related to Nelson's stochastic mechanics. The Riemannian and symplectic structures of the density manifold are studied, and the Schrödinger equation is derived from a variational principle. By a theorem of Moser, the density manifold is an infinite dimensional homogeneous space, being the quotient of the group of diffeomorphisms of the underlying base manifold modulo the group of diffeomorphisms which preserve the Riemannian volume. From this structure and symplectic reduction, the quantization procedure is equivalent to Lie-Poisson equations on the dual of a semidirect product Lie algebra. A Poisson map is obtained between the dual of this Lie algebra and the underlying projective Hilbert space.
\end{abstract}

1. Introduction. The configuration space for a physical problem is a differentiable manifold $M$, and the appropriate phase space is the cotangent bundle $T^{*} M$, which is furnished with a canonical symplectic structure. The quantum state space is the complex Hilbert space $\not=L^{2}(\mu)$ divided by the multiplicative action of $\mathbf{C}^{*}$, where $\mu$ is an appropriate measure on $M$. The classical Hamiltonian generates a group of symplectic automorphisms of $T^{*} M$, but does not act naturally on $\not$. The term quantization refers to the problem of establishing a correspondence between the two mathematical frameworks.

Let us briefly adopt the language of categories to discuss this problem in more detail (see [22]). The classical category $C$ consists of symplectic vector bundles and symplectic isomorphisms. The quantum category $\mathcal{Q}$ is made up of complex Hilbert spaces, and unitary operators. The most general quantization problem, therefore, is to determine a functor $f: \mathcal{C} \rightarrow \mathcal{Q}$ which is required to obey some auxiliary conditions. It is well known however, that no such functor exists, if the bracket operations are to be preserved. An alternative problem, therefore, is to obtain an intermediate category $I$ and a pair of functors $C \leftarrow I \rightarrow \mathcal{Q}$. Since the collection of cotangent bundles lies naturally in (the objects of) $C$, a natural choice for $I$ is to consider the collection of smooth manifolds and smooth diffeomorphisms. To determine a functor from $I$ to $\mathcal{Q}$ assume that the manifold $M$ has a distinguished volume element $\mu$ and form the Hilbert space $H=L^{2}(\mu)$. ( $\mu$ is replaced by equivalence classes of measures in the general case.) A quantization procedure may then involve establishing a correspondence between unitary operators on the Hilbert space $H$ associated with the manifold $M$, and Poisson algebras of functions on the

Received by the editors February 2, 1987.

1980 Mathematics Subject Classification (1985 Revision). Primary 58B20, 58F05; Secondary 58D05, $60 \mathrm{H} 07$. 
cotangent bundle $T^{*} M$. We refer to $[\mathbf{1}, \mathbf{2 2}]$ and the references quoted therein for further discussion of the general topic of quantization.

In this paper, we study an additional structure, formally represented by

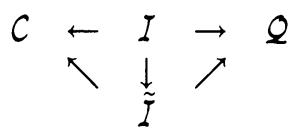

The objects of $\tilde{I}$ consist of density manifolds: if $N$ is an object of $I, N$ maps to $\mathcal{M}(N)$, an infinite dimensional manifold of probability densities on $N$. The cotangent bundle map $\tilde{I} \rightarrow C$ is then naturally defined, and the map $\tilde{I} \rightarrow \mathcal{Q}$ is defined as before by choosing a distinguished density. By studying the symplectic structure of $T^{*} \mathcal{M}(N)$, we provide a classical mathematical framework for quantum theory by passing from finite to infinite dimensional symplectic geometry. Following Nelson [21], we call this procedure configuration space quantization.

Suppose that $M$ and $N$ are $C^{\infty}$ manifolds with distinguished volume elements $d_{M} x$ and $d_{N} x$. If we let $\mathscr{H}(M)=L^{2}\left(d_{M} x\right), \mathcal{H}(N)=L^{2}\left(d_{N} x\right)$, and $\mathscr{H}(M \times N)=$ $L^{2}\left(d_{M} x \times d_{N} x\right)$, we see that there are natural isomorphisms

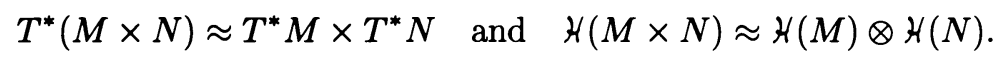

However, the density manifold $\mathcal{M}(M \times N)$ is much larger than the space of product measures $\mathcal{M}(M) \times \mathcal{M}(N)$. We therefore think of $\mathcal{M}(M)$ as a mathematical configuration space and do not assign it interpretational value.

The mathematical structure of configuration space quantization is wholly motivated and guided by stochastic mechanics. In this probabilistic theory, a diffusion is constructed on the physical configuration space $M$, and there is a correspondence between Hamiltonians on the phase space $T^{*} M$ and functionals on the diffusion process. Although the quantization procedure of Markovian stochastic mechanics is derived from a variational principle, the collection of regular Borel measures on path space in this theory does not inherently admit a natural symplectic structure. The present work may therefore be seen as a geometrization of the deterministic relations of stochastic mechanics.

2. Conservative diffusion and stochastic mechanics. In this section we review the basic concepts and constructions of stochastic mechanics in order to motivate and provide technical as well as conceptual background for the following sections.

Let $M$ be an $n$-dimensional $C^{\infty}$ manifold which is a locally compact Hausdorff space, and for an interval $I \subseteq \mathbf{R}$ let the path space $\Omega$ be defined by

$$
\Omega=\prod_{I} \dot{M}
$$

(where $\dot{M}$ denotes the one-point compactification of $M$ ) with the product topology. An element of $\Omega$ is an arbitrary function $\omega: I \rightarrow \dot{M}$. If $B$ denotes the Borel $\sigma$-algebra of $\Omega$ then a regular Borel probability measure $\operatorname{Pr}$ on $(\Omega, B)$ defines an $\dot{M}$-valued stochastic process $\xi(t)_{t \in I}$ over $(\Omega, B, \operatorname{Pr})$ by the evaluation map

$$
\xi(t)(\omega)=\omega(t)
$$

Similarly, an $M$-valued stochastic process over a probability space $(S, S, \mu)$ induces a measure on path space by a procedure developed by Nelson; see [20]. We let 
$P_{t}, \mathcal{F}_{t}$, and $\mathcal{N}_{t}$ denote the $\sigma$-algebras $P_{t}=\sigma\{\xi(s), s \leq t\}, \mathcal{F}_{t}=\sigma\{\xi(s), s \geq t\}$, and $\mathcal{N}_{t}=\sigma\{\xi(s), s=t\}$ generated by $\xi$.

DEFINITION 2.1. We say that $\{\xi(t)\}_{t \in I}$ is a smooth diffusion if

$$
\begin{gathered}
E_{t} d \xi^{i}(t)=E_{t}\left(\xi^{i}(t+d t)-\xi^{i}(t)\right)=\beta^{i}(\xi(t), t) d t+o(d t), \\
d \xi^{i}(t) d \xi^{j}(t)=\sigma^{i j}(\xi(t)) d t+o(d t)
\end{gathered}
$$

in local coordinates $q^{i}$, where $\beta^{i}$ and $\sigma^{i j}$ are smooth functions and $\sigma^{i j}$ is of strictly positive type, and if (2.1) and (2.2) hold for the time-reversed process $\check{\xi}(t)=$ $\xi(-t), t \in I$.

Here $E_{t}$ denotes the conditional expectation with respect to the $\sigma$-algebra $\mathcal{N}_{t}$, and $o(d t)$ is interpreted in the probabilistic sense. To be precise, let $B_{t}$ be the algebra of all uniformly bounded stochastic processes $\left\{\eta_{t}\right\}_{t \in[0, \varepsilon]}$ defined over the same probability space as $\xi$ such that

$$
\left|E_{t} \eta(d t)\right|_{\infty}=O(d t) \text { and }\left|E_{t} \eta(d t)^{2}\right|_{\infty}=O(d t) .
$$

Let $O_{t}$ be the ideal of all $\zeta \in B_{t}$ such that

$$
\left|E_{t} \zeta(d t)\right|_{\infty}=o(d t) \text { and }\left|E_{t} \zeta(d t)^{2}\right|_{\infty}=o(d t) .
$$

Then we write $\alpha(t)=\beta(t)+o(d t)$ in case $\alpha(t)-\beta(t) \in O_{t}$. We assume as part of the definition that

$$
E_{t} d_{*} \xi^{i}(t)=\beta_{*}^{i}(\xi(t), t) d t+o(d t)
$$

and

$$
d_{*} \xi^{i}(t) d_{*} \xi^{j}(t)=\sigma_{*}^{i j}(\xi(t)) d t+o(d t)
$$

for smooth functions $\beta_{*}^{i}$ and $\sigma_{*}^{i j}$ defined in the local chart, where $d_{*} \xi^{i}(t)=\xi^{i}(t)-$ $\xi^{i}(t-d t), d t>0$. The following theorem is an easy consequence of Definition 2.1.

THEOREM 2.2. If $\xi$ is a smooth diffusion and $f \in C_{0}^{\infty}(M \times I)$, the collection of smooth functions with compact support in $M \times I$, then

$$
\begin{gathered}
d \xi^{i} d \xi^{j} d \xi^{k}=o(d t), \\
\lim _{d t \downarrow 0} E_{t} \frac{d f(\xi(t), t)}{d t}=\left(\frac{1}{2} \sigma^{i j} \frac{\partial^{2}}{\partial q^{i} \partial q^{j}}+\beta^{i} \frac{\partial}{\partial q^{i}}+\frac{\partial}{\partial t}\right) f(\xi(t), t), \\
\lim _{d t \downarrow 0} E_{t} \frac{d_{*} f(\xi(t), t)}{d t}=\left(-\frac{1}{2} \sigma_{*}^{i j} \frac{\partial^{2}}{\partial q^{i} \partial q^{j}}+\beta_{*}^{i} \frac{\partial}{\partial q^{i}}+\frac{\partial}{\partial t}\right) f(\xi(t), t)
\end{gathered}
$$

and under a change of coordinates $q^{i} \mapsto q^{i^{\prime}}$

$$
\begin{gathered}
d \xi^{i^{\prime}}=\frac{\partial q^{i^{\prime}}}{\partial q^{i}} d \xi^{i}+\frac{1}{2} \frac{\partial^{2} q^{i^{\prime}}}{\partial q^{i} \partial q^{j}} \sigma^{i j} d t+o(d t) \\
\beta^{i^{\prime}}=\frac{\partial q^{i^{\prime}}}{\partial q^{i}} \beta^{i}+\frac{1}{2} \sigma^{i j} \frac{\partial^{2} q^{i^{\prime}}}{\partial q^{i} \partial q^{j}} \\
\sigma^{i^{\prime} j^{\prime}}=\frac{\partial q^{i^{\prime}}}{\partial q^{i}} \frac{\partial q^{j^{\prime}}}{\partial q^{j}} \sigma^{i j}
\end{gathered}
$$

The point of this theorem is that while $\sigma^{i j}$ is a contravariant 2-tensor which defines a Riemannian metric on $T M, \beta^{i}$ does not transform like a vector and we 
must therefore allow for a correction term in defining the drift vector field of the process $\xi$. This is done by setting

$$
b^{i}=\beta^{i}+\frac{1}{2} \Gamma_{j k}^{i} \sigma^{j k}
$$

where $\Gamma_{j k}^{i}$ are the Christoffel symbols associated with the Riemannian connection for the metric $\sigma$. (Here and throughout we employ the summation convention $\left.A^{i} B_{i}=\sum_{k} A^{k} B_{k}.\right)$ The vector field $b=b^{i}\left(\partial / \partial q^{i}\right)$ is called the forward drift of the process $\xi$. Notice that the choice of metric is intrinsically associated with the diffusion, and determines its quadratic variation.

Now, from the time-symmetric definition of smooth diffusion one can deduce the equality $\sigma^{i j}=\sigma_{*}^{i j}$, and we may therefore define the backward drift vector field $b_{*}=b_{*}^{i}\left(\partial / \partial q^{i}\right)$ by

$$
b_{*}^{i}=\beta_{*}^{i}+\frac{1}{2} \Gamma_{j k}^{i} \sigma^{j k}
$$

using the same connection.

A smooth diffusion $\xi$ has a smooth time-dependent and strictly positive density $\rho$ which satisfies

$$
\int_{M \times I} f d \rho=\int_{I} E[f(\xi(t), t)] d t
$$

for all $f \in C_{0}^{\infty}(M \times I)$. The density $\rho$ therefore satisfies the parabolic forward Fokker-Planck equation

$$
\partial \rho / \partial t=\frac{1}{2} \Delta \rho-\nabla \cdot(b \rho)
$$

(where $\nabla \cdot$ and $\Delta$ are the usual operations on vector fields and scalars associated with the Riemannian metric $\sigma$ ). By introducing the forward and backward stochastic derivatives

$$
\begin{aligned}
& D X(t)=\lim _{d t \downarrow 0} E\left[\frac{d X(t)}{d t} \mid \rho_{t}\right], \\
& D_{*} X(t)=\lim _{d t \downarrow 0} E\left[\frac{d_{*} X(t)}{d t} \mid \mathcal{F}_{t}\right]
\end{aligned}
$$

for a real-valued stochastic process $\{X(t)\}_{t \in I}$ over $(\Omega, B, \operatorname{Pr})$ and utilizing the associated stochastic integration by parts formulae, one can then show that the forward and backward drift vector fields are related by

$$
b_{*}^{i}=b^{i}-\nabla^{i} \log \rho
$$

and, in addition, that $\rho$ satisfies the antiparabolic backward Fokker-Planck equation

$$
\partial \rho / \partial t=-\frac{1}{2} \Delta \rho-\nabla \cdot\left(b_{*} \rho\right)
$$

We therefore have that the continuity equation (or current equation)

$$
\partial \rho / \partial t=-\nabla \cdot(v \rho)
$$

and the osmotic equation

$$
u^{i}=\frac{1}{2}\left(\nabla^{i} \rho / \rho\right)
$$

hold, when we define the current velocity by $v=\frac{1}{2}\left(b+b_{*}\right)$ and the osmotic velocity by $u=\frac{1}{2}\left(b-b_{*}\right)$. 
By differentiating (2.11) in time and using (2.10) we find that

$$
\partial u / \partial t=-\nabla\left(\frac{1}{2} \nabla \cdot v+u \cdot v\right) .
$$

The time evolution of the current velocity, however, is not as easily obtained from the basic relations governing the infinitesimal characteristics of $\xi$. In the context of stochastic mechanics, $\partial v / \partial t$ is obtained from a stochastic variational principle.

Notice that if $f \in C_{0}^{\infty}(M \times I)$, then the stochastic derivative $D f(\xi(t), t)$ is given by

$$
D f(\xi(t), t)=\frac{1}{2} \Delta f(\xi(t), t)+b \cdot \nabla f(\xi(t), t)+\frac{\partial f(\xi(t), t)}{\partial t} .
$$

We call $\frac{1}{2} \Delta+b \cdot \nabla$ the forward diffusion operator, and the analogous backward diffusion operator is given by $-\frac{1}{2} \Delta+b_{*} \cdot \nabla$. To extend the stochastic derivatives to the full tensor algebra requires a stochastic parallel translation map, which we denote by $\tau$. The correct definition of this map in the context of stochastic mechanics was given by Dohrn and Guerra (see [20]). It is obtained by applying a deterministic translation map to the geodesic approximation of the diffusion, as follows. Let $\left\{\eta(s) \mid s \in\left[s_{0}, s_{1}\right]\right\}$ be a geodesic on $M$ with $v(s)=d \eta / d s$ the vector field tangent to $\eta$ and let $u_{0} \in T_{\eta\left(s_{0}\right)} M$. Then $u(s)=\tau\left(\eta(s), \eta\left(s_{0}\right)\right) u_{0}$ is defined to be the Jacobi field along $\eta$ with initial condition $\nabla_{v} u\left(s_{0}\right)=0$; that is, $u(s)$ satisfies

$$
D^{2} u / d s+R(u, v) u=0
$$

with initial conditions

$$
D u / d s=0, \quad u\left(s_{0}\right)=u_{0}
$$

where $D / d s$ denotes the covariant derivative along $\eta$, and $R$ is the Riemannian curvature tensor. It is an elementary fact that every Jacobi field may be obtained by a variation through geodesics. To construct this variation through geodesics for $\tau$, let $\left\{\gamma\left(s_{0}, t\right) \mid t \in[0,1]\right\}$ be a curve satisfying $\gamma\left(s_{0}, 0\right)=\eta\left(s_{0}\right), \partial \gamma\left(s_{0}, t\right) /\left.\partial t\right|_{t=0}=u_{0}$, and let $\left\{\gamma(s, t) \mid s \in\left[s_{0}, s_{1}\right]\right\}$ be the geodesic beginnning at $\gamma\left(s_{0}, t\right)$, with initial velocity $\bar{\tau}\left(\eta\left(s_{0}\right), \gamma\left(s_{0}, t\right)\right) v\left(s_{0}\right)$, where $\bar{\tau}$ denotes the Levi-Civita parallel translation. It is then easy to check that

$$
u(s)=\partial \gamma(s, t) /\left.\partial t\right|_{t=0}
$$

is the Jacobi field described by (2.13) and (2.14). If one now takes the geodesic approximation to the diffusion $\xi$ and applies $\tau$ to each geodesic segment, the stochastic differential equation

$$
d Y^{i}=-\Gamma_{k l}^{i} Y^{k} d \xi^{l}-\frac{1}{2} \sigma^{k l} Y^{m} d t\left(\Gamma_{k l}^{j} \Gamma_{m j}^{i}-\Gamma_{j l}^{i} \Gamma_{m k}^{j}-\Gamma_{k j}^{i} \Gamma_{m l}^{j}+\frac{\partial}{\partial q^{m}} \Gamma_{k l}^{i}\right)
$$

is obtained, which may be represented intrinsically by the Stratanovich stochastic differential equation

$$
d Y^{i}=-\Gamma_{k l}^{i} Y^{k} \circ d X^{l}+\frac{1}{2} R_{j k l}^{i} Y^{l} \sigma^{j k} d t .
$$

For a tensor field $a$, the stochastic derivatives $D a$ and $D_{*} a$ are then defined by setting

$$
D a=\lim _{d t \downarrow 0} \frac{1}{d t} E_{t}[\tau(\xi(t), \xi(t+d t)) a(\xi(t+d t), t+d t)-a(\xi(t), t)]
$$


and

$$
D_{*} a=\lim _{d t \downarrow 0} \frac{1}{d t} E_{t}[a(\xi(t), t)-\tau(\xi(t), \xi(t-d t)) a(\xi(t-d t), t-d t)]
$$

which leads to the relations

$$
\begin{gathered}
D a(\xi(t), t)=\left(\frac{1}{2} \Delta_{\mathrm{DG}}+b \cdot \nabla+\partial / \partial t\right) a(\xi(t), t), \\
D_{*} a(\xi(t), t)=\left(-\frac{1}{2} \Delta_{\mathrm{DG}}+b_{*} \cdot \nabla+\partial / \partial t\right) a(\xi(t), t) .
\end{gathered}
$$

The second order operator $\Delta_{\mathrm{DG}}$ is the Dohrn-Guerra Laplacian $\Delta_{\mathrm{DG}}=\nabla^{i} \nabla_{i}-R_{\text {: }}$, with $R$ : denoting the induced action of the Ricci tensor $R$ on the mixed tensor algebra $\mathrm{T}(M)$. The Laplacian $\Delta_{D G}$ agrees with the de Rham-Kodaira Laplacian on scalars and 1-forms.

2.1 Stochastic action. From Theorem 2.2 we see that the stochastic increment $d \xi^{i}(t)=\xi^{i}(t+d t)-\xi^{i}(t)$ does not transform like a vector. To obtain a vector quantity naturally associated with $d \xi^{i}$ we make use of the exponential map of the Riemannian manifold $M$. To this end, define $\tilde{d} \xi \in T_{\xi(t)} M$ by

$$
\exp _{\tilde{d} \xi}(1)=\exp _{\tilde{d} \xi / d t}(d t)=\xi(t+d t) \text {. }
$$

Since exp is a local isomorphism, $\tilde{d} \xi$ is (almost surely) well-defined when $d t$ is sufficiently small, and

$$
E_{t} \tilde{d} \xi=b(\xi(t), t) d t+o(d t) .
$$

Let $\{\eta(s) \mid t \leq s \leq t+d t\}$ be the minimal geodesic joining $\xi(t)$ and $\xi(t+d t)$. Then

and

$$
d \eta /\left.d s\right|_{s=t}=\tilde{d} \xi / d t
$$

$$
\left(\int_{t}^{t+d t}\left|\frac{d \eta}{d s}\right| d s\right)^{2}=\left(\int_{t}^{t+d t} \sqrt{\left\langle\frac{d \eta}{d s}, \frac{d \eta}{d s}\right\rangle} d s\right)^{2}
$$

is the square of the Riemannian distance between $\xi(t)$ and $\xi(t+d t)$. However, since

$$
\left(\int_{t}^{t+d t} \sqrt{\left\langle\frac{d \eta}{d s}, \frac{d \eta}{d s}\right\rangle} d s\right)^{2}=\left\langle\frac{d \eta}{d s}, \frac{d \eta}{d s}\right\rangle d t^{2}+o\left(d t^{2}\right)=\langle\tilde{d} \xi, \tilde{d} \xi\rangle+o\left(d t^{2}\right)
$$

we would like to estimate $E_{t}\langle\tilde{d} \xi, \tilde{d} \xi\rangle$ to $o\left(d t^{2}\right)$ in order to provide an approximation for $E[\langle d \xi / d t, d \xi / d t\rangle]$. The point of this calculation would be that although the paths of $\xi$ are almost surely nowhere differentiable, if the divergent quantity $\lim _{d t \downarrow 0} \sum_{n} E\left[\left\langle d \xi\left(t_{n}\right) / d t, d \xi\left(t_{n}\right) / d t\right\rangle\right] d t$ may be given a well-defined meaning and expressed in terms of the infinitesimal characteristics of the diffusion $\xi$, then it will serve as the classical action of $\xi$ and should determine the appropriate Lagrangian for the quantization procedure of stochastic mechanics. This idea is realized in the following theorem of Guerra and Nelson.

THEOREM 2.3. Let $\{\xi(t)\}_{t \in I}$ be a smooth Markovian diffusion on $M$ with forward drift vector field $b$, and fix $t \in I$. Let $d t>0$ be sufficiently small so that

$$
\tilde{d} \xi=\exp ^{-1}(\xi(t+d t)) \in T_{\xi(t)} M
$$

is almost surely well-defined. Then

$$
\frac{1}{2} E_{t}\left\langle\frac{\tilde{d} \xi}{d t}, \frac{\tilde{d} \xi}{d t}\right\rangle=\frac{1}{2}\langle b, b\rangle+\frac{1}{2} \nabla \cdot b+\frac{1}{12} \bar{R}+\frac{n}{2 d t}+o(1)
$$

where $\bar{R}$ is the scalar curvature of the metric induced by $\xi$. 
The important aspect of this result is that the expression (2.15) is renormalizable in the following sense. The divergent term $n / 2 d t \rightarrow \infty$ is deterministic; it does not depend on the particular path, and therefore drops out upon taking variations of the diffusion $\xi$ by processes of identical quadratic variation.

The expression (2.15) for the classical action of a Markovian diffusion leads to the following notion of criticality. Let $\phi \in C_{0}^{\infty}(M)$ and let $L: T M \times I \rightarrow \mathbf{R}$ be the Lagrangian on $T M$ given by

$$
L(v, t)=\frac{1}{2}\langle v, v\rangle_{\pi(v)}-\phi(\pi(v), t)
$$

where, as before, $x \mapsto\langle\cdot, \cdot\rangle_{x}$ denotes the Riemannian metric on $T M$ and $\pi: T M \rightarrow$ $M$ is the canonical projection. If $\{\xi(t)\}_{t \in I}$ is a smooth Markovian diffusion with forward drift vector field $b$ and diffusion tensor giving the metric $\langle\cdot, \cdot\rangle$, we may form the associated Lagrangian $L_{b}: M \times I \rightarrow \mathbf{R}$ by setting

$$
L_{b}(x, t)=\left(\frac{1}{2}\langle b, b\rangle+\frac{1}{2} \nabla \cdot b+\frac{1}{12} \bar{R}-\phi\right)(x, t) .
$$

The associated action is

$$
I\left[b, t_{0}, t_{1}\right]=E\left[\int_{I} L_{b}(\xi(t), t) d t\right]=\int_{M} \int_{I} L_{b}(x, t) \rho(x, t) d t
$$

where $I=\left[t_{0}, t_{1}\right]$. We may also re-express $L_{b}$ in the following form, which will be especially convenient for our later developments.

PROPOSITION 2.4. Let $\xi$ be a smooth diffusion with forward drift vector field $b$, backward drift $b_{*}$, and density $\rho$. Then

$$
\begin{aligned}
E\left[L_{b}(\xi(t), t)\right] & =E\left[\left(\frac{1}{2}\langle v, v\rangle-\frac{1}{2}\langle u, u\rangle-\phi+\frac{1}{12} \bar{R}\right)(\xi(t), t)\right] \\
& =\int_{M}\left(\frac{1}{2}\langle v, v\rangle-\frac{1}{2}\langle u, u\rangle-\phi+\frac{1}{12} \bar{R}\right)(x, t) \rho(x, t) d_{M} x
\end{aligned}
$$

where $v=\frac{1}{2}\left(b+b_{*}\right)$ and $u=\frac{1}{2}\left(b-b_{*}\right)=\frac{1}{2}(\nabla \rho / \rho)$ are respectively the current velocity and osmotic velocity vector fields of the diffusion $\xi$.

PROOF. By partial integration we see that since $\rho>0$,

$$
\frac{1}{2} \int_{M} \nabla \cdot b \rho=-\int_{M}\left\langle b, \frac{1}{2} \frac{\nabla \rho}{\rho}\right\rangle \rho=-\int_{M}\langle b, u\rangle \rho .
$$

But $\frac{1}{2}\langle b, b\rangle-\langle b, u\rangle=\frac{1}{2}\langle v, v\rangle-\frac{1}{2}\langle u, u\rangle$, and since the contributions from $\phi$ and $\bar{R}$ are identical in equations (2.17) and (2.18), the result follows.

REMARK. Notice that we may simply hide the scalar curvature term in the Lagrangian by setting $\phi_{\bar{R}}=\phi-\frac{1}{12} \bar{R}$ and considering the Lagrangian $L_{\bar{R}}(v, t)=$ $\frac{1}{2}\langle v, v\rangle-\phi_{\bar{R}}(\pi(v), t)$. We will therefore not include this term in the following expressions; the interested reader may refer to [20] and the references quoted therein for a discussion of this Pauli-DeWitt term.

DEFINITION 2.5. The smooth Markovian diffusion $\xi$ is said to be critical for the Lagrangian $L$ if for each interval $\left[t, t_{1}\right] \subseteq I=\left[t_{0}, t_{1}\right]$ and every smooth vector field $\delta b$ with compact support in $M \times\left[t, t_{1}\right]$ we have that

$$
I\left[b, t, t_{1}\right]-I^{\prime}\left[b^{\prime}, t, t_{1}\right]=o(\delta b)
$$


where

$$
I^{\prime}\left[b^{\prime}, t, t_{1}\right]=E^{\prime}\left[\int_{t}^{t_{1}} L_{b+\delta b}\left(\xi^{\prime}(s), s\right) d s\right] .
$$

Here $E^{\prime}$ denotes the expectation with respect to the measure of the smooth Markovian diffusion $\xi^{\prime}$ having forward drift vector field $b^{\prime}=b+\delta b$, and the same diffusion tensor and probability density at time $t$ as $\xi(t)$.

The following theorem is due to Guerra and Morato, and serves to specify the dynamics of a critical diffusion. The crucial step in the Guerra-Morato variational principle is the introduction of the stochastic analogue of Hamilton's principle function, which is the scalar given by

$$
S(\xi(r), r)=-E\left[\int_{r}^{t_{1}} L_{b}(\xi(s), s) d s \mid \xi(r)\right]
$$

and the expansion of the stochastic derivatives of this random variable with respect to both the critical and "variational" processes. The approach given below is more probabilistic, and assumes less regularity of the principal function than the method which treats the stochastic differentiation operations as second order partial differential operators.

THEOREM 2.6. Suppose that $S$ is defined as in (2.19), that the gradient $\nabla S$ exists in $L^{2}(\rho)$, and that

$$
d S(\xi(t), t)=\nabla S(\xi(t), t) \cdot d w(t)+O(d t),
$$

where the $O(d t)$ term is $\xi(t)$-measurable, and $w$ is the underlying Wiener process. Then the smooth Markovian diffusion $\xi$ is critical for $L$ given by (2.16) if and only if the current velocity $v$ of $\xi$ satisfies the stochastic Hamilton-Jacobi condition

$$
v(x, t)=\nabla S(x, t) \text {. }
$$

PROOF. As described above, let $\xi^{\prime}$ be the Markovian diffusion with forward drift vector field $b^{\prime}=b+\delta b$. Let

$$
M(r)=\exp \left(\int_{t}^{r}\langle\delta b(\xi(s), s), d w(s)\rangle+\frac{1}{2} \int_{t}^{r}\|\delta b(\xi(s), s)\|^{2} d s\right)
$$

be the Girsanov density associated with $\xi^{\prime}$ (see [12]). Since

$$
I=E\left[\int_{t}^{t_{1}} L_{b}(\xi(s), s) d s\right]=E[S(\xi(t), t)]=E[S(\xi(t), t) M(t)]
$$

and $S\left(\xi\left(t_{1}\right), t_{1}\right)=0$ almost surely, we may use the algebraic identity

$$
d(S(\xi(s), s) M(s))=d S(\xi(s), s) M(s)+S(\xi(s), s) d M(s)+d S(\xi(s), s) d M(s)
$$

to obtain an expression for $I$ by stochastic integration by parts. Because $M$ is a $P_{t}$-martingale there will be no contribution from the $S(\xi(s), s) d M(s)$ term under expectation. Furthermore, by expanding the exponential and again using the martingale property it is easy to see that

$$
\begin{aligned}
E[d S(\xi(s), s) d M(s)] & =E\left[\nabla_{i} S d \xi^{i}(s) M(s)\left(\int_{s}^{s+d s} \delta b^{i} d w_{i}(s)+o(\delta b)\right)\right] \\
& =E\left[\nabla_{i} S\left(b^{i} d s+d w^{i}\right) M(s) \int_{s}^{s+d s} \delta b^{i} d w_{i}\right]+o(\delta b)+o(d s) \\
& =E\left[\nabla_{i} S \delta b^{i} M(s) d s\right]+o(\delta b)+o(d s) .
\end{aligned}
$$


We therefore have that

$$
-I=E\left[\int_{t}^{t_{1}} D S(\xi(s, s)) M(s) d s\right]+E\left[\int_{t}^{t_{1}} \nabla_{i} S \delta b^{i}(\xi(s), s) M(s) d s\right]+o(\delta b) .
$$

Now, from the Markov property it is clear that

$$
D S=L_{b}
$$

and, similarly, that $D^{\prime} S^{\prime}=L_{b}^{\prime}$. (Note that this remains true for non-Markovian processes when the stochastic derivatives are taken with respect to the past filtrations.) Expanding the Lagrangians in $\delta b$ yields

$$
L_{b^{\prime}}-L_{b}=\langle b, \delta b\rangle+\frac{1}{2} \nabla \cdot \delta b+o(\delta b) .
$$

Finally, using the relations

$$
\frac{1}{2} E^{\prime}[\nabla \cdot \delta b]=\frac{1}{2} \int \nabla \cdot \delta b \rho+o(\delta b)=-E[\langle\delta b, u\rangle]+o(\delta b)
$$

and $b-u=v$, by combining the above expressions, we arrive at

$$
\begin{aligned}
I^{\prime}-I & =E\left[\int_{t}^{t_{1}}\langle v-\nabla S, \delta b\rangle(\xi(s), s) M(s) d s\right]+o(\delta b) \\
& =E\left[\int_{t}^{t_{1}}\langle v-\nabla S, \delta b\rangle\left(\xi^{\prime}(s), s\right) d s\right]+o(\delta b) .
\end{aligned}
$$

The result now follows since $\delta b$ is only restricted to have compact support in $M \times$ $\left[t, t_{1}\right]$.

COROLlARY 2.7. Let $\xi$ be critical for $L$ and let $S$ be defined by (2.1). Then the stochastic Hamilton-Jacobi equation

$$
\frac{\partial S}{\partial t}+\frac{1}{2}\langle\nabla S, \nabla S\rangle+\phi-\frac{1}{2}\langle u, u\rangle-\frac{1}{2} \nabla \cdot u=0
$$

holds.

ProOF. By combining the relation (2.23) with (2.21) we obtain

$$
\frac{\partial S}{\partial t}+b \cdot \nabla S+\frac{1}{2} \Delta S=\frac{\partial S}{\partial t}+\langle b, v\rangle+\frac{1}{2} \nabla \cdot v=\frac{1}{2}\langle b, b\rangle+\nabla \cdot b-\phi .
$$

(Recall the above remark regarding the scalar curvature.) Using the relation $v+u=$ $b$ then yields equation (2.24).

COROLLARY 2.8. The diffusion $\xi$ is critical for $L$ iff the Schrödinger equation

$$
i \frac{\partial \psi}{\partial t}=-\frac{1}{2} \Delta+\phi \psi
$$

holds for $\psi$ given by $\psi=e^{R+i S}$, where $u=\nabla R$ and $v=\nabla S$ are the osmotic and current velocity vector fields of $\xi$, and $S$ is given as in (2.20).

Proof. Combining the stochastic Hamilton-Jacobi equation (2.24) with the current equation (2.10) expressed in terms of the scalars $R$ and $S$ results in the nonlinear system

$$
\frac{\partial S}{\partial t}+\frac{1}{2}\langle\nabla S, \nabla S\rangle+\phi-\frac{1}{2}\langle\nabla R, \nabla R\rangle-\frac{1}{2} \Delta R=0
$$




$$
\frac{\partial R}{\partial t}+\langle\nabla R, \nabla S\rangle+\frac{1}{2} \Delta S=0 \text {. }
$$

A simple computation then shows that the above system is equivalent to the Schrödinger equation (2.25).

REMARK. We note that a covector term may be included in the above Lagrangians as well. See [20] and $\S 5$.

2.2 Paths of density. There are several ways of constructing a smooth Markovian diffusion on $M$. Given a collection of vector fields one can construct a stochastic flow of diffeomorphisms of the frame bundle and project onto $M$. The familiar method of taking discrete approximations to Itô stochastic integral equations yields a diffusion locally, and the resulting local processes in different charts may be glued together. A partial differential equations approach begins with an initial density $\rho_{0}$ and the drift vector field $b(\cdot, t), t \in I$, and solves the forward diffusion equation to obtain the probability transition function $p$, and therefore the measure Pr on path space.

From the point of view of the present paper, it is most natural to suppose that for each $t \in I, \rho(\cdot, t)$ is a smooth strictly positive probability density on the compact manifold $M$. The osmotic velocity, determined by

$$
u=\frac{1}{2} \nabla \log \rho,
$$

is smooth and we may define the current velocity by the relation

$$
v=-\frac{1}{\rho} \nabla \Delta^{-1} \frac{\partial \rho}{\partial t}
$$

which exists since $\int \partial \rho / \partial t=0$ implies $\partial \rho / \partial t \in \operatorname{Ran}(\Delta)$. Then $\rho$ satisfies the forward and backward diffusion equations (associated with $b=v+u$ and $b_{*}=v-u$ ) which have fundamental solutions $p$ and $p_{*}$. The measure on path space may then be generated using the transition kernel

$$
\begin{aligned}
\rho\left(d x_{1}, t_{1} ; \ldots ; d x_{n}, t_{n}\right)= & p_{*}\left(d x_{1}, t_{1} ; x_{2}, t_{2}\right) \cdots p_{*}\left(d x_{i-1}, t_{i-1} ; x_{i}, t_{i}\right) \rho\left(d x_{i}, t_{i}\right) \\
& \cdot p\left(x_{i}, t_{i} ; d x_{i+1}, t_{i+1}\right) \cdots p\left(x_{n-1}, t_{n-1} ; d x_{n}, t_{n}\right) .
\end{aligned}
$$

Thus, a smooth path of densities may be used to generate a Markovian diffusion.

The following theorem, due to Carlen [5], provides the proper mathematical setting for stochastic mechanics.

THEOREM 2.9. For each $t \in\left[t_{0}, t_{1}\right]=I$ let $\rho(\cdot, t)$ be a probability density, and let $u$ and $v$ be time-dependent vector fields on $M$ which satisfy

$$
\int_{M} \int_{I}(\langle v, v\rangle+\langle u, u\rangle) \rho(x, t) d_{M} x d t<\infty
$$

and

$$
\int_{M} f(x) \rho(x, t) d_{M} x-\int_{M} f(x) \rho(x, s) d_{M} x=\int_{s}^{t} \int_{M}\langle v, \nabla f\rangle \rho(x, r) d_{M} x d r
$$

for all $s, t \in I$ and $f \in C_{0}^{\infty}(M)$. Then there is a regular probability measure $\operatorname{Pr}$ on path space $\Omega$ such that under $\operatorname{Pr}, t \mapsto \xi(t)$ is a square integrable Markov process with density $\rho(\cdot, t)$, and the following limits exist strongly in $L^{2}(\operatorname{Pr})$ for any $f \in C_{0}^{\infty}(M)$ :

$$
\begin{aligned}
& \lim _{d t \downarrow 0} \frac{1}{d t} E\left[f(\xi(t+d t))-f(\xi(t)) \mid \mathcal{N}_{t}\right]=\left(\frac{1}{2} \Delta+b \cdot \nabla\right) f(\xi(t)), \\
& \lim _{d t \downarrow 0} E\left[f(\xi(t))-f(\xi(t-d t)) \mid \mathcal{N}_{t}\right]=\left(-\frac{1}{2} \Delta+b_{*} \cdot \nabla\right) f(\xi(t))
\end{aligned}
$$

where $b=v+u$ and $b_{*}=v-u$ are the forward and backward drift vector fields. 
The relevance of the finite action condition (2.28) and the weak continuity equation (2.29) for stochastic quantization is contained in the following result.

THEOREM 2.10. Let $\phi$ be a Rellich class potential on $M \times I$ and let $\psi_{0}$ satisfy $|\psi|_{2}^{2}=\int_{M}\|\nabla \psi\|^{2} d_{M} x<\infty$. Then if $\psi_{t}=\psi(\cdot, t)$ is a solution of the Schrödinger equation (2.25) with potential $\phi$ and initial condition $\psi_{0}$ (that is, if $\psi_{t}=$ $\exp (-i t H) \psi_{0}$ for the Hamiltonian $\left.H=-\frac{1}{2} \Delta+\phi\right)$, then $u, v$, and $\rho$ defined by

$$
\begin{aligned}
& u(x, t)= \begin{cases}\mathfrak{R} e \frac{\nabla \psi(x, t)}{\psi(x, t)}, & \psi(x, t) \neq 0, \\
0, & \psi(x, t)=0,\end{cases} \\
& v(x, t)= \begin{cases}\Im m \frac{\nabla \psi(x, t)}{\psi(x, t)}, & \psi(x, t) \neq 0, \\
0, & \psi(x, t)=0,\end{cases}
\end{aligned}
$$

and

$$
\rho(x, t)=|\psi(x, t)|^{2}
$$

satisfy the finite action condition (2.28) and the weak continuity equation (2.29).

We refer to [5] for the proofs of these results. We end this section by briefly discussing the interpretational aspect of Markovian stochastic mechanics. Through the study of some particular quantum systems, it has been determined that Markovian stochastic mechanics violates a certain separability property which one may justifiably demand of a physically tenable theory. This is essentially due to the fundamental property that a component of a multidimensional Markov process is not again, in general, a Markov process - the generation of $\sigma$-algebras does not respect vector space structure. (Consider the sheet component of a Wiener process on the Riemann surface of $\sqrt{z}$.) However, it is not at all clear how the GuerraMorato variational principle may be extended to non-Markovian diffusions, or that such an extension would remove the separability problem.* Thus, from the point of view of Nelson, one should turn toward the construction of random fields on physical space-time rather than diffusions on configuration space. See $[\mathbf{2 0}, \mathbf{2 1}]$ for a treatment of the locality problem in stochastic mechanics. The point to be made here is that there is no mention of Markovicity or other restrictions in specifying a path of densities $\rho$. Hence, if a quantization theory can be formulated using paths of density as the fundamental mathematical objects of consideration, then there may be no questions of locality or separability directly involved. We carry out this program in the following sections.

3. The density manifold. Throughout this section, $M$ will denote a $C^{\infty}$, compact, connected, Riemannian $n$-manifold without boundary. A volume element is a positive $n$-form of odd kind, in the sense of de Rham. We let $\mathcal{M}=\mathcal{M}(M)$ denote the collection of smooth $\left(=C^{\infty}\right)$ densities on $M$. To be precise, let $\mathcal{N}$ be

${ }^{*}$ One approach to this problem uses the homogeneous chaos decomposition of Wiener space as a basis for a variational principle for non-Markovian semimartingales. 
the vector space of sections of the bundle of smooth $n$-forms over $M$, and let $\mathcal{K} \subseteq \mathcal{N}$ be the closed subspace

$$
\mathcal{K}=\left\{\eta \in \mathcal{N} \mid \int_{M} \eta=0\right\}
$$

Consider the Riemannian volume element $d_{M} x=\mu$, form the closed affine subspace $\bar{K}=K+\mu$, and set

$$
\mathcal{M}=\{\rho \in \bar{K} \mid \rho=f \mu \text { locally, with } f>0\} .
$$

We may assume, of course, that $\operatorname{Vol}(M)=\int_{M} \mu=1$, so that if $\rho \in \mathcal{M}$, then $\int_{M} \rho=1$. Thus, $\mathcal{M}$ is an open convex subset of a closed affine subspace of the vector space $\mathcal{N}$.

In this section we study the manifold structure of $\mathcal{M}$ and briefly discuss its relationship with the group $D(M)$ of diffeomorphisms of $M$. This relationship is further developed in $\S 6$. Consider the following simple example, which gives an indication of the interplay between $\mathcal{M}$ and $D$. Let $M=S^{1}$, and let $D\left(S^{1}\right)$ denote the group of orientation preserving diffeomorphisms of $S^{1}$. If $\eta \in D\left(S^{1}\right)$, then $\eta$ lifts naturally to a real-valued periodic function (also denoted $\eta$ ) on $\mathbf{R}$. Clearly $\eta^{\prime}$ is a density, and an exercise in calculus shows that $D\left(S^{1}\right) \approx \mathcal{M}\left(S^{1}\right) \times \mathbf{R} / 2 \pi$. Therefore $D\left(S^{1}\right)$ has the same homotopy type as $S^{1}$, since $\mathcal{M}$ is contractible. (This is the trivial case of the Smale conjecture: $D\left(S^{n}\right)$ is homotopically equivalent to $\mathrm{SO}(n+1), n=1,2,3,4$. The case $n=4$ appears to be open.)

This discussion of the relation between the diffeomorphism group and the density manifold in 3.3 and 3.4 will lead to a treatment of the Fréchet manifold structure of $\mathcal{M}$ and its tangent bundle $T \mathcal{M}$ in 3.5 through 3.7. In particular, in 3.5 we develop the fundamental properties of the osmotic Laplacian $\Delta_{\rho}$, which is essential to many further results. This operator is used to study the geometric structure of $\mathcal{M}$ in 3.6 and 3.7.

3.1 Calculus on Fréchet manifolds. We will be working in the $C^{\infty}$ category, and therefore briefly recall here some basic facts concerning the Fréchet calculus.

By a grading on the Fréchet space $X$ we mean a family of seminorms $\{|\cdot|\}_{n \in \mathbf{N}}$ which generate the topology of $X$, and which satisfies $|\cdot|_{0} \leq|\cdot|_{1} \leq \cdots \leq|\cdot|_{n} \leq \cdots$. For example, let $V$ be a finite-dimensional vector bundle over $M$, and consider the space $C^{\infty}(M, V)$ of smooth sections. Let $U_{\alpha}$ be an open cover of $M$ and let $\phi: U_{\alpha} \rightarrow V_{\alpha}$ be local trivializations. For each $\alpha$, let $K_{\alpha} \subseteq V_{\alpha}$ be compact, and set $\bar{K}_{\alpha}=\phi_{\alpha}^{-1}\left(K_{\alpha}\right)$. Also, assume that

$$
\nabla: C^{\infty}(M, V) \rightarrow C^{\infty}\left(M, T^{*} M \otimes V\right)
$$

is a bundle connection. Define the family of seminorms $\left\{|\cdot|_{K_{\alpha}, n}^{\alpha}\right\}$ by

$$
|f|_{K_{\alpha}, n}^{\alpha}=\sum_{j=1}^{n} \sum_{i_{1}, \ldots, i_{j}} \sup _{\bar{K}_{\alpha}}\left|\nabla_{i_{1}} \nabla_{i_{2}} \cdots \nabla_{i_{j}} f(x)\right|
$$

where the indices $i_{1}, \ldots, i_{n}$ refer to a basis for $\left.T M\right|_{U_{\alpha}}$, and $\nabla_{j}$ is the covariant derivative for $\nabla$. By restricting to a countable subcover (or finite in the compact case) and letting a countable collection of compact sets fill out each coordinate chart, it is then evident that the above family of seminorms generates a grading for a Fréchet topology on $C^{\infty}(M, V)$ which is independent of the local trivializations 
chosen. In the same fashion one constructs a grading which is compatible with the topology generated by the Sobolev seminorms.

Although there is no canonical extension of Banach space differential calculus to Fréchet spaces, the following provides a good working definition.

DEFINITION 3.1. Let $X$ and $Y$ be Fréchet spaces. Suppose that $U \subseteq X$ is open, and that $f: U \rightarrow Y$ is a continuous map. If $x \in U$ and $v \in X$, the directional derivative of $f$ at $x$ in the direction $v$ is defined to be the element $d f_{x}(v)$ of $Y$ given by

$$
d f_{x}(v)=\lim _{t \rightarrow 0} \frac{f(x+t v)-f(x)}{t}
$$

if this limit exists.

Since the vector space $\mathcal{L}(X, Y)=\operatorname{Hom}(X, Y)$ is not, in general, a Fréchet space when $X$ and $Y$ are Fréchet the usual notion of $C^{1}$ Fréchet derivative in Banach spaces, namely, that the mapping $x \mapsto d f_{x}$ is continuous in the norm topology of $\mathcal{L}(X, Y)$, does not apply. The following weaker criterion is therefore adopted in Fréchet spaces.

DEFINITION 3.2. The map $f: U \subseteq X \rightarrow Y$ is said to be of class $C^{1}$ in case the limit $d f_{x}(v)$ always exists, and the mapping given by $d f: U \times X \rightarrow Y, d f(x, v)=$ $d f_{x}(v)$ is jointly continuous as a function of two variables. Higher derivatives and the class $C^{k}, k \geq 2$, are defined in the obvious inductive fashion.

Within this framework the basic operations of the calculus are sufficiently well behaved for our purposes. In particular, the notion of Fréchet manifold, defined in the obvious way, is well adapted to our study of $\mathcal{M}$. We refer to $[11,17]$ for further discussion of the general theory.

REMARK. Apart from certain topological and analytical issues, a large part of the finite-dimensional geometric theory carries over unchanged to the infinitedimensional case when it is formulated in an appropriate algebraic fashion. In [19] the theory is cast in the abstract setting of Lie modules, which provides a suitable framework for much of what we shall carry out in later sections.

3.2 The group of diffeomorphisms. We now quickly review the basic facts concerning the group of diffeomorphisms of the compact manifold $M$. For details and many further developments we refer to [11] and [17], where the $C^{\infty}$ point of view is developed, and to [2] and [6], where the Hilbert manifold case is developed.

Let $D(M)$ denote the group of $C^{\infty}$ diffeomorphisms of $M$, with the $C^{\infty}$ topology. The tangent space $T_{e} D(M)$ at the identity $e$ is identified with equivalence classes of smooth curves of diffeomorphisms through $e$. Thus the Lie algebra $\mathcal{L}(D(M))$ consists of the collection $X(M)$ of smooth vector fields $x \mapsto V(x) \in T_{x} M$ with the $C^{\infty}$ topology.

A one-parameter subgroup of $D(M)$ is simply the flow of a vector field $V \in$ $\chi(M)$, and this flow is defined for all time since $M$ is compact. We therefore have a map EXP: $\chi(M) \rightarrow D(M)$ which takes $V \in \chi(M)$ to the one-parameter group of diffeomorphisms $\operatorname{EXP}(t V)$ satisfying $d \gamma / d t=V(\gamma), \gamma(0)=x_{0}$, when we set $\gamma(t)=\operatorname{EXP}(t V)\left(x_{0}\right)$. Unlike the situation with finite-dimensional Lie groups, however, this exponential map does not provide a local chart structure on $D(M)$ since it is not a local isomorphism-it does not map onto any neighborhood of the identity. Some more work must therefore be done to provide an atlas for $D(M)$. 
The idea behind providing a chart structure for $D(M)$ may be thought of as follows. Let $V_{\varepsilon} \subseteq \chi(M)$ be a neighborhood of zero which satisfies $|v|<\varepsilon$ for all $v \in V_{\varepsilon}$, where $|\cdot|$ denotes the Riemannian norm on $T M$. Choose $\varepsilon$ sufficiently small so that any two points of $M$ with Riemannian distance less than $\varepsilon$ may be joined by a unique geodesic of length less than $\varepsilon$, and such that this geodesic is minimal and depends smoothly on its endpoints. (For example, if $M=S^{n}, \varepsilon<\pi$ will do.) Let exp denote the exponential map of $M$. Then for $v \in V_{\varepsilon}$ the $\operatorname{map} \phi(v): M \rightarrow M$ given by $\phi(v)(x)=\exp _{v(x)}(1)$ may be thought of as mapping $x \in M$ to a geodesic of length $|v|<\varepsilon$, and $\phi: V_{\varepsilon} \rightarrow C^{\infty}(M, M)$ is a homeomorphism onto an open set $U_{\varepsilon} \subseteq C^{\infty}(M, M)$. Define $\bar{U}_{\varepsilon}$ by

$$
\bar{U}_{\varepsilon}=\left\{\eta \in U_{\varepsilon} \mid \eta \text { is a diffeomorphism }\right\} .
$$

Then $\bar{V}_{\varepsilon}=\phi^{-1}\left(\bar{U}_{\varepsilon}\right)$ is open in $V_{\varepsilon}$. This provides a local chart $\phi: \bar{V}_{\varepsilon} \rightarrow \bar{U}_{\varepsilon}$ at the identity. A local chart structure for all of $D(M)$ is then obtained by using the group action. This provides $D(M)$ with the structure of a smooth Fréchet manifold modelled on the Fréchet space $\chi(M)$. The Lie product of the Lie algebra $T_{\varepsilon} D(M) \approx X(M)$ is minus the usual bracket of vector fields

$$
[X, Y]^{i}=X^{j} \frac{\partial}{\partial q^{j}} Y^{i}-Y^{j} \frac{\partial}{\partial q^{j}} X^{i} .
$$

The minus sign comes from the fact that one works with right- rather than leftinvariant vector fields.

Now the group $T D(M)$ may be expresed as a semidirect product

$$
T D(M) \approx D(M) \ltimes_{\gamma} T_{e} D(M),
$$

where $T_{e} D(M)$ is a normal subgroup. In particular, we have that the fiber $T_{\eta} D(M)$, for $\eta \in D(M)$, is given by

$$
T_{\eta} D(M)=\{V: M \rightarrow T M \mid \pi V(x)=\eta(x)\} .
$$

Thus, $T_{\eta} D(M)$ is the collection of smooth vector fields over $\eta$, represented by the commutative diagram

$$
\begin{aligned}
& T M \\
& M \stackrel{V \nearrow}{\stackrel{\eta}{\rightarrow}} \quad M .
\end{aligned}
$$

We denote the group actions associated with $\eta$ by $R_{\eta}$ and $L_{\eta}$ :

$$
\begin{array}{ll}
L_{\eta}: D(M) \rightarrow D(M), & \xi \mapsto L_{\eta} \xi=\eta \circ \xi, \\
R_{\eta}: D(M) \rightarrow D(M), & \xi \mapsto R_{\eta} \xi=\xi \circ \eta .
\end{array}
$$

The induced actions on $T D(M)$ are

$$
\begin{array}{ll}
T L_{\eta}: T D(M) \rightarrow T D(M), & V_{\xi} \in T_{\xi} D(M) \mapsto T L_{\eta} V_{\xi}=T \eta \circ V_{\xi} \in T_{\eta \circ \xi} D(M), \\
T R_{\eta}: T D(M) \rightarrow T D(M), & V_{\xi} \in T_{\xi} D(M) \mapsto T R_{\eta} V_{\xi}=V_{\xi} \circ \eta \in T_{\xi \circ \eta} D(M),
\end{array}
$$

and these maps are smooth. The adjoint action Ad is therefore given by pushforward of vector fields:

$$
\operatorname{Ad}_{\eta}: T_{e} D(M) \rightarrow T_{e} D(M), \quad V \mapsto \operatorname{Ad}_{\eta} V=T_{e}\left(L_{\eta} \circ R_{\eta^{-1}}\right) V=\eta_{*} V .
$$

REMARK. From these relations it is clear that when $D(M)$ is modelled on $H^{s}$, it is not a Lie group since the group operations are not smooth. 
Two important subgroups of $D(M)$ are the group of volume preserving diffeomorphisms

$$
D_{\mu}(M)=\left\{\eta \in D(M) \mid \eta^{*} \mu=\mu\right\},
$$

and the group of symplectic diffeomorphisms

$$
D_{\omega}(M)=\left\{\eta \in D(M) \mid \eta^{*} \omega=\omega\right\}
$$

in the case that $M$ admits a symplectic structure $\omega$. To show that $D_{\mu}(M)$ is a closed smooth submanifold of $D(M)$ one considers the cohomology class

$$
[\mu]=\mu+d\left(C^{\infty}\left(\Lambda^{n-1}(M)\right)\right)
$$

(where $C^{\infty}\left(\Lambda^{k}(M)\right)$ denotes the smooth exterior $k$-forms), and shows that the mapping $F: D(M) \rightarrow[\mu]$ described by $F(\eta)=\eta^{*} \mu$, is a submersion. Then $D_{\mu}(M)=$ $F^{-1}(\mu)$ is a smooth submanifold, but this only determines the local chart structure of $D_{\mu}(M)$ implicitly.

The proof of this fact is similar to that of the following theorem, which is central to the developments of $\S 6$.

THEOREM 3.3. The Lie group $D(M)$ acts transitively on $\mathcal{M}$ by pullback of forms. Furthermore, $\pi: D(M) \rightarrow \mathcal{M}$ is a principal fiber bundle with structure group $D_{\mu}(M)$ and projection $\pi \eta=\eta^{*} \mu$.

For a proof of this theorem, which is indicative of the methodology of diffeomorphism groups, we refer to [6]. The proof is based on a beautiful (and now standard) technique due to Moser [18], which generalizes not only to the Darboux theorem, but to Weinstein's theorem on normal forms for Lagrangian submanifolds. The proof given in [11] uses heat equation and inverse function theorem methods.

A density is thus a coset of diffeomorphisms. The Lie algebra $\mathcal{L}\left(D_{\mu}(M)\right)$ of the Lie group $D_{\mu}(M)$ is the Fréchet space $X_{\mu}(M)=\{X \in \chi(M) \mid \nabla \cdot X=0\}$, the space of vector fields which are divergence-free with respect to the measure $\mu$. This follows from the observation that if $t \mapsto \eta_{t}$ is a curve in $D_{\mu}(M)$ with $X_{t}=d \eta / d t \in T_{\eta_{t}} D_{\mu}(M)$, then

$$
0=\frac{d}{d t} \eta_{t}^{*} \mu=\eta_{t}^{*}\left(L_{X_{t} \circ \eta_{t}^{-1}} \mu\right)
$$

and thus $\nabla \cdot\left(X_{t} \circ \eta_{t}^{-1}\right)=0$. The tangent space $T_{\mu} \mathcal{M}$ should therefore be identified with $\mathcal{G} \chi(M)$, the gradient vector fields on $M$, since by the Hodge decomposition

$$
\mathcal{L}(D(M)) \approx \chi(M)=\chi_{\mu}(M) \oplus \mathcal{G} \chi(M) .
$$

In other words, $\pi: D(M) \rightarrow \mathcal{M}$ is a submersion, and we have

PROPOSITION 3.4. There is a short exact sequence of Fréchet spaces given by

$$
0 \rightarrow T_{e} D_{\mu}(M) \rightarrow T_{e} D(M) \rightarrow T_{\mu} \mathcal{M} \rightarrow 0
$$

which splits canonically.

In the next two sections we will establish a more concrete description of this relationship, and, in particular, we shall see how the splitting of this sequence provides an important feature of the density manifold for our later developments.

3.3 The osmotic Laplacian. There is nothing special about the volume form $\mu$ in the above discussion. Indeed, we may let $\rho_{0} \in \mathcal{M}$ be an initial density, and for 
$t \mapsto \eta_{t}$ a path in $D(M)$ with $\eta_{0}=e$, let $\rho_{t}=\left(\eta_{t}\right)_{*} \rho_{0}$ be the push-forward density path. Then

$$
0=\frac{d}{d t}\left(\eta_{t}^{*} \rho_{t}\right)=\eta_{t}^{*}\left(L_{X_{t} \circ \eta_{t}^{-1}} \rho_{t}+\frac{d \rho_{t}}{d t}\right)
$$

holds, where $X_{t} \in T_{\eta_{t}} D(M)$ is the vector field over $\eta_{t}$ tangent to $t \mapsto \eta_{t}$. Thus, under the mapping $\rho_{t} \mapsto \rho_{t} / \mu$ from $n$-forms to smooth, strictly positive functions, the continuity equation

$$
\partial \rho / \partial t+\nabla \cdot\left(x_{t} \rho_{t}\right)=0
$$

holds when we set $x_{t} \circ \eta_{t}=X_{t}$. Of course, the same equation holds if we replace $x_{t}$ by $x_{t}+z_{t}$, where $z_{t}$ is a vector field on $M$ having divergence zero with respect to the measure $\rho_{t}\left(\approx \rho_{t} d_{M} x\right)$.

With $\rho=\rho_{0}$, suppose that we seek to minimize the energy

$$
\frac{1}{2} \int_{M}\|x\|^{2} \rho=\frac{1}{2}\left\langle\langle v, v\rangle_{\rho}\right.
$$

among all vector fields $v$ which satisfy the continuity equation for $\rho(t)$ at $t=0$. If $v$ is the unique vector field which minimizes (3.3) then $v$ is orthogonal to all $\rho$-divergence-free vector fields with respect to the inner product $\langle\langle\cdot, \cdot\rangle\rangle_{\rho}$. Thus, since $\rho>0, v^{b}$ is a closed 1 -form. Without assumption on the $H^{1}(M, \mathbf{R})$, we would like to show that $v^{b}$ is exact $[\mathbf{2 1}]$.

THEOREM 3.5. Let $t \mapsto \rho(t)$ be a smooth curve in $\mathcal{M}$ with $\rho(0)=\rho$. Then there is a function $S \in C^{\infty}(M)$, dejined uniquely up to a constant, such that

$$
\partial \rho /\left.\partial t\right|_{t=0}=-\nabla \cdot(\nabla S \rho)
$$

ProOF. For the purposes of this proof, let $\not$ be the complex Hilbert space $L^{2}\left(\rho d_{M} x\right)$ with inner product $\langle\cdot, \cdot\rangle_{0}$,

$$
\langle f, g\rangle_{0}=\int_{M} f \bar{g} \rho d_{M} x,
$$

and let $\nvdash^{1}$ be the completion of $C^{\infty}(M)$ with respect to the bilinear form $\langle\cdot, \cdot\rangle_{1}$ :

$$
\langle f, g\rangle_{1}=\frac{1}{2} \int_{M}\langle\nabla f, \overline{\nabla g}\rangle \rho d_{M} x+\int_{M} f \bar{g} \rho d_{M} x .
$$

Clearly $\nvdash^{1}$ is a dense linear subspace of $\not$, and is a Hilbert space with larger norm $|f|_{1} \geq|f|_{0}, f \in \mathcal{H}^{1}$. Thus, it follows that there is a unique positive selfadjoint operator associated with the quadratic form $\langle\cdot, \cdot\rangle_{1}$ with form domain $\mathfrak{H}^{1}$. If we denote this operator by $-\frac{1}{2} \Delta_{\rho}$, then

$$
\langle f, g\rangle_{1}=\left\langle f,-\frac{1}{2} \Delta_{\rho} g\right\rangle_{0}+\langle f, g\rangle_{0}
$$

for $f \in \mathcal{H}^{1}$ and $g \in \operatorname{Dom}\left(-\frac{1}{2} \Delta_{\rho}\right)$. Furthermore, for $g \in \operatorname{Dom}\left(-\frac{1}{2} \Delta_{\rho}\right)$ we have that

$$
\frac{1}{2} \Delta_{\rho} g=\frac{1}{2} \Delta g+\frac{1}{2} \frac{\nabla \rho}{\rho} \cdot \nabla g=\frac{1}{2} \Delta g+u \cdot \nabla g .
$$

Let $\nvdash_{0} \subseteq \not{H}$ be the closed subspace $\mathfrak{H}_{0}=\left\{f \in \not{H} \mid \int_{M} f \rho=0\right\}$. Then $\frac{1}{2} \Delta_{\rho}$ is an elliptic operator with discrete spectrum, and kernel consisting of the constants, which maps $\nvdash_{0}$ into itself. It also has a bounded inverse $\left(\frac{1}{2} \Delta_{\rho}\right)^{-1}$. 
Now, clearly $\rho^{-1}(\partial \rho / \partial t) \in \mathscr{H}_{0}$ since

$$
\int_{M}\left(\frac{1}{\rho} \frac{\partial \rho}{\partial t}\right) \rho=\frac{d}{d t} \int_{M} \rho=0 .
$$

Thus,

$$
S=\left(\frac{1}{2} \Delta_{\rho}\right)^{-1}\left(-\frac{1}{2 \rho} \frac{\partial \rho}{\partial t}\right)
$$

exists in $\varkappa_{0}$, and by elliptic regularity is $C^{\infty} . S$ is the desired solution (up to a constant) of (3.4).

COROLLARY 3.6. Let $\rho \in \mathcal{M}$. We may write

$$
\chi(M)=g(M) \oplus X_{\rho}(M),
$$

where $\mathcal{G} X(M)$ is the collection of gradient vector fields on $M$, and $\chi_{\rho}(M)$ is orthogonal to $\mathcal{G} X(M)$ with respect to the bilinear form

on $X(M) \times X(M)$.

$$
(X, Y) \mapsto\left\langle\langle X, Y\rangle_{\rho}=\int_{M}\langle X, Y\rangle \rho\right.
$$

PROOF. If $z \in \mathcal{X}(M)$ we may consider a smooth curve $t \mapsto \rho(t)$ in $\mathcal{M}$ with

$$
\partial \rho /\left.\partial t\right|_{t=0}=-\nabla \cdot(z \rho) \text {. }
$$

The operator $\frac{1}{2} \Delta_{\rho}$ then provides a smooth gradient vector field

$$
Q(\rho) z=-\nabla\left(\frac{1}{2} \Delta_{\rho}^{-1}\right)\left(\frac{1}{2 \rho} \frac{\partial \rho}{\partial t}\right)
$$

and since $P(\rho) z=z-Q(\rho) z$ satisfies $\nabla \cdot(P(\rho) z \rho)=0$, it follows that $P(\rho) z$ and $Q(\rho) z$ are orthogonal with respect to $\left\langle\langle\cdot, \cdot\rangle_{\rho}\right.$.

We call the operator $\Delta_{\rho}$ constructed in Theorem 3.5 the osmotic Laplacian. It will play an important role in what follows. The advantage of the above construction is that it goes through in the more general case where $M$ is not compact and the density $\rho$ may have nodes.

PROPOSITION 3.7. The collection $\left\{\Delta_{\rho}\right\}_{\rho \in \mathcal{M}}$ is a smooth one-parameter family of elliptic operators with corresponding smooth family of inverses $\left\{\Delta_{\rho}^{-1}\right\}_{\rho \in \mathcal{M}}$.

ProOF. Consider the grading on $C^{\infty}(M)$ generated by the Sobolev norms

$$
|f|_{n}=\sum_{|\alpha| \leq n}\left|\nabla^{\alpha} f\right|
$$

with $|\cdot|$ the $L^{2}\left(d_{M} x\right)$ norm, as in the construction of $\S 2.2$. By Sobolev embedding, this is equivalent to the $C^{n}$ grading. From general elliptic theory we have that if the mapping

$$
f \mapsto L(f)=\sum_{|\alpha| \leq r} f_{\alpha} \cdot D^{\alpha}, \quad f \in U,
$$

defines a family of elliptic operators of degree $r$ (where generally the coefficients $f=\left(f_{\alpha}\right)$ are sections of a jet bundle) then for $g \in U$, if $|f-g|_{0}<\varepsilon$ one obtains estimates of the form

$$
|h|_{n+r} \leq C\left(|k|_{n}+|f|_{n}|k|_{0}\right)
$$


where $L(f) h=k$. For $\Delta_{\rho}$, we require that the osmotic velocities be close,

$$
\left\|\nabla \log \left(\rho / \rho_{0}\right)\right\|_{0}<\varepsilon
$$

to obtain estimates of the form

$$
\left|\Delta_{\rho}^{-1} k\right|_{n+2} \leq C\left(|k|_{n}+|\nabla \log \rho|_{n}|k|_{0}\right) .
$$

REMARK. In considering osmotic diffusion where $\partial \rho / \partial t=0$, the operator $\frac{1}{2} \Delta_{\rho}$ may be used to construct a measure on path space yielding a symmetric Markov process. Recent work has been carried out by D. Bakry and M. Emery [4] on Sobolev and logarithmic Sobolev inequalities related to $\Delta_{\rho}$ on noncompact manifolds.

3.4 The Fréchet manifold structure of $\mathcal{M}$. Notice that the appearance of the Lie derivative in the expression

$$
\eta_{t}^{*}\left(L_{X_{t} \circ \eta_{t}^{-1}} \rho_{t}+d \rho_{t} / d t\right)=0
$$

which is equivalent to the continuity equation, transforms the tangent $X_{t}$ to the path of diffeomorphisms into an implicit tangent vector $x_{t}=X_{t} \circ \eta_{t}^{-1}$ for the path of densities by the relation $\partial \rho / \partial t+\nabla \cdot(x \rho)=0$. We now provide $\mathcal{M}$ with a chart structure which makes this correspondence explicit.

THEOREM 3.8. Let $\rho \in \mathcal{M}$ and let $V_{\rho}$ be a neighborhood of zero in $\mathcal{K}=\{f \in$ $\left.C^{\infty}(M) \mid \int_{M} f d_{M} x=0\right\}$ such that $\Delta_{\rho} S<1$ for all $S \in V_{\rho}$. Let $\phi_{\rho}: V_{\rho} \rightarrow \mathcal{M}$ be the mapping given by $\phi_{\rho}(S)=\rho-\nabla \cdot(\nabla S \rho)$. Then the collection of such pairs $\left\{V_{\rho}, \phi_{\rho}\right\}_{\rho \in \mathcal{M}}$ forms a $C^{\infty}$ atlas on $\mathcal{M}$.

Proof. Let $S \in V_{\rho}$. Since $\Delta_{\rho} S<1$ we see that $\phi_{\rho}(S)=\rho-\nabla \cdot(\nabla S \rho)=$ $\rho\left(1-\Delta_{\rho} S\right)>0$. In addition, since $\int_{M}\left(\Delta_{\rho} S\right) \rho=0$, it follows that $\phi_{\rho}$ maps $V_{\rho}$ into $\mathcal{M}$, as claimed.

Recall that a closed subspace of a Fréchet space is again Fréchet. The mapping $S \mapsto \phi_{\rho}(S)-\rho=-\left(\Delta_{\rho} S\right) \rho$ is a linear, invertible map of $\mathcal{K}$ into itself. Since $(\rho, S) \mapsto \Delta_{\rho} S$ is smooth, it follows from the open mapping theorem that the set $\phi_{\rho}\left(V_{\rho}\right)-\rho$ is open in $\mathcal{K}$. Thus, $\phi_{\rho}\left(V_{\rho}\right)$ is open in $\mathcal{M}$.

Let $\rho_{1}, \rho_{2} \in \mathcal{M}$ with corresponding charts $\phi_{i}: V_{i} \rightarrow U_{i} \subseteq \mathcal{M}, i=1,2$, and consider the composition

$$
\phi=\phi_{1}^{-1} \circ \phi_{2}: \phi_{2}^{-1}\left(U_{1} \cap U_{2}\right) \rightarrow \phi_{1}^{-1}\left(U_{1} \cap U_{2}\right) .
$$

If $\rho=\rho_{1}-\nabla \cdot\left(\nabla S_{1} \rho_{1}\right)=\rho_{2}-\nabla \cdot\left(\nabla S_{2} \rho_{2}\right) \in U_{1} \cap U_{2}$, then $\left(\Delta_{\rho_{1}} S_{1}\right) \rho_{1}=\rho_{1}-\rho_{2}+$ $\left(\Delta_{\rho_{2}} S_{2}\right) \rho_{2}$, so that

$$
\begin{aligned}
S_{1} & =\phi\left(S_{2}\right)=\Delta_{\rho_{1}}^{-1}\left(1-\frac{\rho_{2}}{\rho_{1}}+\left(\Delta_{\rho_{2}}\right) \frac{\rho_{2}}{\rho_{1}}\right) \\
& =\Delta_{\rho_{1}}^{-1}\left(1-\frac{\rho_{2}}{\rho_{1}}\right)+\Delta_{\rho_{1}}^{-1}\left(\left(\Delta_{\rho_{2}} S_{2}\right) \frac{\rho_{2}}{\rho_{1}}\right),
\end{aligned}
$$

noticing that $\left(1-\rho_{2} / \rho_{1}\right),\left(\Delta_{\rho_{2}} S_{2}\right) \rho_{2} / \rho_{1} \in \mathcal{K}_{\rho_{1}}=\left\{f \mid \int f \rho_{1}=0\right\}$. From our previous remarks regarding the osmotic Laplacian it now follows that $\phi$ is smooth.

Notice that if $t \mapsto \rho(t)$ is a curve through $\rho(0)=\rho$ in $\mathcal{M}$, then $\partial \rho / \partial t$ makes sense as a Gâteux derivative. We therefore think of the gradient vector field

$$
v=-\nabla\left(\frac{1}{2} \Delta_{\rho}^{-1}\right)\left(\frac{1}{2 \rho} \frac{\partial \rho}{\partial t}\right) \in T_{\rho} \mathcal{M}
$$


as an intrinsic tangent vector (or an intrinsic representation) at $\rho$ since there is no mention of local charts. A vector field on $\mathcal{M}$ is thus a smooth (as a map between Fréchet spaces) assignment $\rho \mapsto X(\rho)$ of a gradient vector field $X(\rho) \in \mathcal{G} X(M)$ to each $\rho \in \mathcal{M}$.

A simple consequence of the chart structure provided by Theorem 3.8 is the following, which provides the most natural framework for working with tensors on $\mathcal{M}$.

PROPOSITION 3.9. Let $X_{1}, \ldots, X_{n}$ be vector fields on $\mathcal{M}$ and let $\rho \in \mathcal{M}$. Then there is a chart $\phi: V \subseteq \mathcal{G} X(M) \rightarrow U \subseteq \mathcal{M}$ with $\rho \in \mathcal{M}$, such that

$$
\left.\frac{\partial}{\partial t} \phi \circ \rho_{i}(t)\right|_{t=0}=X_{i}(\rho), \quad i=1, \ldots, n,
$$

where for each $i, t \mapsto \rho_{i}(t)$ is a curve representing $X_{i}$ at $\rho$. In other words, the intrinsic and local representations of $X_{i}$ agree at $\rho$.

Proof. Let $\varepsilon>0$ be such that the set $\bar{V}=\left\{t X_{i}(\rho) \mid t \in(-\varepsilon, \varepsilon), i=1, \ldots, n\right\}$ satisfies $\rho-\nabla \cdot(v \rho)>0$ for all $v \in \bar{V}$. Extend $\bar{V}$ to an open neighborhood $V \subseteq \mathcal{G} X(M)$ such that again $\rho-\nabla \cdot(v \rho)>0$ for $v \in V$. Now define $\phi: V \rightarrow \mathcal{M}$ by $\phi(v)=\rho-\nabla \cdot(v \rho)$. For $i=1, \ldots, n$ let $t \mapsto \rho_{i}(t)$ represent $X_{i}$ at $\rho$. Then

$$
\phi^{-1} \circ \rho_{i}(t)=\phi^{-1} \circ\left(\rho-t \nabla \cdot\left(X_{i}(\rho) \rho\right)\right)+o(d t)=t X_{i}(\rho)+o(d t)
$$

so that

$$
\left.\frac{\partial}{\partial t} \phi^{-1} \circ \rho_{i}(t)\right|_{t=0}=X_{i}(\rho)
$$

for each $i$.

Finally, it will be useful to think of tangent vectors on $\mathcal{M}$ as equivalence classes of vector fields on $M$, as in the fiber bundle description of Theorem 3.3. To this end we make the following definition.

DEFINITION 3.10. For each $\rho \in \mathcal{M}$, let $\equiv_{\rho}$ denote the relation on $\mathcal{X}(M) \times \mathcal{X}(M)$ defined by $X \equiv_{\rho} Y$ in case $\nabla \cdot((X-Y) \rho)=0$. Then $\equiv_{\rho}$ is an equivalence relation on $X(M)$ and by Theorem 3.5 there is a unique gradient vector field within each equivalence class. We denote by $Q(\rho): \chi(M) \rightarrow \chi(M)$ the projection operator which takes $X \in \mathcal{X}(M)$ to the unique gradient vector field $Q(\rho) X$ in the equivalence class $[X]_{\rho}$ containing $X$. For $\rho \in \mathcal{M}$ let $\mathcal{V}_{\rho}$ be the collection of equivalence classes of vector fields on $M$ under the equivalence relation $\equiv_{\rho}$. It is then easy to prove that $\mathcal{V}=\cup_{\rho} \mathcal{V}_{\rho}$ forms a vector bundle over $\mathcal{M}$ with projection $\pi_{\nu}$, such that the following diagram commutes:

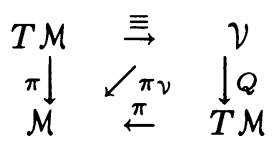

Thus, the $\rho$-equivalence class

$$
[v]_{\rho}=\left[\nabla\left(\frac{1}{2} \Delta_{\rho}\right)^{-1}\left(\frac{-1}{2 \rho} \frac{\partial \rho}{\partial t}\right)\right]_{\rho}
$$

is associated with the path $t \mapsto \rho(t) \in \mathcal{M}$. 
Together with the results of $\S 2.4$ then, we may summarize the relationship between $D(M), D_{\mu}(M)$, and $\mathcal{M}$ in the following commutative diagram:

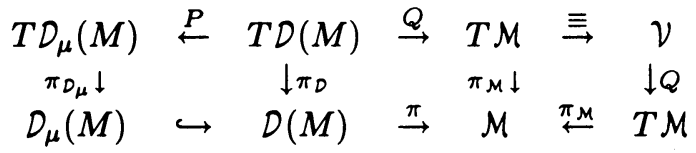

3.5 The tangent bundle $T \mathcal{M}$. Let $X$ and $Y$ be vector fields on $\mathcal{M}$. For each $\rho \in \mathcal{M}, X(\rho) \in T_{\rho} \mathcal{M} \approx \mathcal{G} X(M)$ is a gradient vector field. Let $\left\langle\langle\cdot, \cdot\rangle_{\rho}\right.$ be the inner product

$$
\left\langle\langle X, Y\rangle_{\rho}=\int_{M}\langle X(\rho), Y(\rho)\rangle \rho(x) d_{M} x .\right.
$$

The mapping $\rho \mapsto\left\langle\langle\cdot, \cdot\rangle_{\rho}\right.$ determines a weak Riemannian structure on $T \mathcal{M}$. The term weak is used since the topology generated on $T_{\rho} \mathcal{M}$ by the bilinear form $\left\langle\langle\cdot, \cdot\rangle_{\rho}\right.$ is strictly weaker than the topology inherited from $\mathcal{M}$. Thus, each fiber $T_{\rho} \mathcal{M}$ is a pre-Hilbert space in the $\left\langle\langle\cdot, \cdot\rangle_{\rho}\right.$ inner product.

DEFINITION 3.11. Let $t \mapsto \rho(t) \in \mathcal{M}$ be a curve representing $X$ at $\rho \in \mathcal{M}$. Interpreting $Y: \mathcal{M} \rightarrow \mathcal{G} X(M)$ as a map between Fréchet spaces we define $X \circ Y(\rho) \in$ $T_{\rho} \mathcal{M}$ by

$$
X \circ Y(\rho)=\left.\frac{\partial}{\partial t} Y(\rho(t))\right|_{t=0}=d Y_{\rho}(-\nabla \cdot(X(\rho) \rho))
$$

To determine the Lie product on $T M$ we need the following lemma.

LEMMA 3.12. Let $X \equiv \nabla S_{X}$ and $Y \equiv \nabla S_{Y}$ be vector fields on $\mathcal{M}$. Then

$$
[X(\rho), Y(\rho)] \equiv_{\rho}\left(\Delta_{\rho} S_{Y}\right) X-\left(\Delta_{\rho} S_{X}\right) Y .
$$

ProOF. Clearly this result only depends on $X$ and $Y$ at $\rho$. We need to show that

$$
\left\langle\left\langle Z,\left(\Delta_{\rho} S_{Y}\right) X-\left(\Delta_{\rho} S_{X}\right) Y\right\rangle_{\rho}=\langle\langle Z,[X(\rho), Y(\rho)]\rangle \rho\right.
$$

for any $Z \in \mathcal{G} \mathcal{X}(M)$. Since the Riemannian connection on $T M$ is torsion-free, integrating $\left\langle\langle Z,[X, Y]\rangle_{\rho}\right.$ by parts yields

$$
\begin{aligned}
\int_{M}\left\langle Z, \nabla_{X} Y-\nabla_{Y} X\right\rangle \rho= & \int_{M} Z^{i}\left(X^{j} \nabla_{j} Y_{i}-Y^{j} \nabla_{j} X_{i}\right) \rho \\
= & \int_{M}\left(Y^{j} \nabla_{j} Z^{i} X_{i}-X^{j} \nabla_{j} Z^{i} Y_{i}\right) \rho \\
& +\int_{M}\left(Z^{i} X_{i} \nabla_{j}\left(Y^{j} \rho\right)-Z^{i} Y_{i} \nabla_{j}\left(X^{j} \rho\right)\right) .
\end{aligned}
$$

But the second integral above reduces to $\int_{M}\left(Z^{i} X_{i} \Delta_{\rho} S_{Y}-Z^{i} Y_{i} \Delta_{\rho} S_{X}\right) \rho$, and thus

$$
\left\langle\langle Z,[X, Y]\rangle_{\rho}=\left\langle\left\langle\nabla_{Y} Z, X\right\rangle_{\rho}-\left\langle\left\langle\nabla_{X} Z, Y\right\rangle_{\rho}+\left\langle\left\langle Z,\left(\Delta_{\rho} S_{Y}\right) X-\left(\Delta_{\rho} S_{X}\right) Y\right]\right\rangle\right\rangle_{\rho} .\right.\right.
$$

Now, since $Z$ is a gradient vector field on $M, Y^{j} \nabla_{j} Z^{i} X_{i}=Y^{j} \nabla^{i} Z_{j} X_{i}=\left\langle Y, \nabla_{X} Z\right\rangle$. Therefore we obtain

$$
\left\langle\langle Z,[X, Y]\rangle_{\rho}=\left\langle\left\langle Z,\left(\Delta_{\rho} S_{Y}\right) X-\left(\Delta_{\rho} S_{X}\right) Y\right\rangle\right\rangle_{\rho}\right.
$$

as desired. 
It is instructive to verify this directly. This is accomplished by checking the identity

$$
\begin{gathered}
\nabla_{i}\left(\left(X^{k} \nabla_{k} Y^{i}-Y^{k} \nabla_{k} X^{i}\right) \rho\right)+\nabla_{i}\left(\left(\nabla_{k} X^{k}+\frac{\nabla_{k} \rho}{\rho} X^{k}\right) Y^{i} \rho\right) \\
-\nabla_{i}\left(\left(\nabla_{k} Y^{k}+\frac{\nabla_{k} \rho}{\rho} Y^{k}\right) X^{i} \rho\right)=0
\end{gathered}
$$

with the aid of the Ricci identity.

THEOREM 3.13. Let $X$ and $Y$ be vector fields on $\mathcal{M}$, and let $\llbracket X, Y \rrbracket$ be the Lie product of $X$ and $Y$. Then for each $\rho \in \mathcal{M}$,

$$
\llbracket X, y \rrbracket(\rho) \equiv \rho X \circ Y(\rho)-Y \circ X(\rho)+[X(\rho), Y(\rho)] \text {. }
$$

ProOF. By the Hahn-Banach theorem it suffices to determine the action of $\llbracket X, Y \rrbracket$ as a derivation. Thus, let $f \in C^{\infty}(\mathcal{M})$ be a smooth scalar. Then

$$
\llbracket X, Y \rrbracket f(\rho)=(X(Y(f))-Y(X(f)))(\rho)
$$

canonically defines the Lie product. If $d f_{\rho}$ denotes the directional derivative of $f$ at $\rho$ as a mapping between Fréchet spaces, then this may be expressed as

$$
\llbracket X, Y \rrbracket f(\rho)=X\left(d f_{\rho}(-\nabla \cdot(Y(\rho) \rho))(\rho)\right)-Y\left(d f_{\rho}(-\nabla \cdot(X(\rho) \rho))(\rho)\right) .
$$

Next, taking a second derivative along integral curves of $X$ and $Y$ we obtain

$$
\begin{aligned}
\llbracket X, Y \rrbracket f(\rho)= & d^{2} f_{\rho}(-\nabla \cdot(Y(\rho) \rho),-\nabla \cdot(X(\rho) \rho)) \\
& +d f_{\rho}\left(-\nabla \cdot\left(X \circ Y(\rho) \rho-\Delta_{\rho} S_{X} Y(\rho) \rho\right)\right) \\
& -d^{2} f_{\rho}(-\nabla \cdot(X(\rho) \rho),-\nabla \cdot(Y(\rho) \rho)) \\
& -d f_{\rho}\left(-\nabla \cdot\left(Y \circ X(\rho) \rho-\Delta_{\rho} S_{Y} X(\rho) \rho\right)\right) .
\end{aligned}
$$

Now $d^{2} f_{\rho}$ is a symmetric bilinear operator, so the $d^{2} f$ terms cancel. The above expression thus reduces to

$$
\llbracket X, Y \rrbracket f(\rho)=d f_{\rho}\left(-\nabla \cdot\left(\left(X \circ Y(\rho)-Y \circ X(\rho)+\left(\Delta_{\rho} S_{Y}\right) X-\left(\Delta_{\rho} S_{X}\right) Y\right) \rho\right)\right)
$$

and then from Lemma 3.12 to

$$
\llbracket X, Y \rrbracket f(\rho)=d f_{\rho}(-\nabla \cdot((X \circ Y(\rho)-Y \circ X(\rho)+[X(\rho), Y(\rho)]) \rho)) .
$$

Therefore, $\llbracket X, Y \rrbracket \equiv X \circ Y-Y \circ X+[X, Y]$, as claimed.

Of course, the Jacobi identity follows immediately from the definition of the Lie product as a bilinear, antisymmetric mapping on derivations of the associative ring of scalars. However, since $\mathcal{G} X(M)$ is not closed as an algebra under the bracket $[\cdot, \cdot]$ on $T M$, the Jacobi identity for the bracket $\llbracket \cdot, \cdot]$ is not immediately clear from the expression (3.11). This result is thus most naturally interpreted in the bundle $\pi_{\nu}: \mathcal{V} \rightarrow \mathcal{M}$. To verify the Jacobi identity directly, consider the equivalence class $\left[\sum_{\hookrightarrow} \llbracket[X, Y \rrbracket, Z \rrbracket]_{\rho}\right.$ where $\sum_{\hookrightarrow}$ denotes cyclic summation. A calculation shows that

$$
\sum_{\hookrightarrow} \llbracket \llbracket X, Y \rrbracket, Z \rrbracket(\rho) \equiv \rho \sum_{\hookrightarrow}([Q(\rho)[X, Y], Z]-Z \circ Q(\rho)[X, Y])
$$

where we may assume that $X, Y$, and $Z$ are gradient vector fields which are independent of $\rho$. Now, in general, we have

$$
d_{\rho}\left(\Delta_{\rho} g(\rho)\right)(v)=\Delta_{\rho} d g_{\rho}(v)+\nabla g \cdot d_{\rho}\left(\frac{\nabla \rho}{\rho}\right)(v)
$$


so that letting $g=\Delta_{\rho}^{-1} f$ we obtain

$$
d_{\rho}\left(\Delta_{\rho}^{-1} f\right)(v)=\Delta_{\rho}^{-1}\left(d_{\rho} f(v)-\nabla \Delta_{\rho}^{-1} f \cdot d_{\rho}\left(\frac{\nabla \rho}{\rho}\right)(v)\right) .
$$

Setting $f=\rho^{-1} \nabla \cdot([X, Y] \rho)$, a calculation then shows that

$$
\begin{aligned}
d_{\rho}(Q(\rho)[X, Y])(Z) & =d_{\rho}\left(-\nabla \Delta_{\rho}^{-1}\left(\frac{1}{\rho} \nabla \cdot([X, Y] \rho)\right)\right)(Z) \\
& =Q(\rho)\left(\Delta_{\rho} S_{Z}(Q(\rho)[X, Y]-[X, Y])\right) \\
& \equiv \rho \Delta_{\rho} S_{Z}(Q(\rho)[X, Y]-[X, Y]) .
\end{aligned}
$$

Therefore

$$
\sum_{\hookrightarrow} \llbracket \llbracket X, Y \rrbracket, Z \rrbracket \equiv \sum_{\hookrightarrow}\left([Q[X, Y], Z]+\Delta_{\rho} S_{Z}([X, Y]-Q[X, Y])\right) .
$$

Taking the $\rho$-divergence of both sides, and using $L_{[X, Y]}=\left[L_{X}, L_{Y}\right]$, with $L_{X} \mu=$ $\nabla_{\mu} X \mu$ yields

$$
\begin{aligned}
\sum_{\hookrightarrow} \nabla \cdot(\llbracket \llbracket X, Y \rrbracket, Z \rrbracket \rho)= & \sum_{\hookrightarrow}(Q[X, Y] \cdot \nabla \nabla \cdot(Z \rho)-Z \cdot \nabla \nabla \cdot([X, Y] \rho)) \\
& +\sum_{\hookrightarrow}([X, Y]-Q[X, Y]) \cdot \nabla \nabla \cdot(Z \rho) \\
= & 0,
\end{aligned}
$$

proving that the Jacobi identity holds with respect to the equivalence relation $\equiv$.

In considering the Jacobi identity for $T \mathcal{M}$, recall the following definition. A homogeneous space $G / H$ is said to be reductive if there exists a linear subspace $m$ of $\mathfrak{g}$, the Lie algebra of $G$, such that

$$
\mathfrak{g}=\mathfrak{h} \oplus \mathfrak{m}
$$

and $\operatorname{Ad}_{H} \mathfrak{m} \subseteq \mathfrak{m}$, where $\mathfrak{h}$ is the Lie algebra of $H$. If $p$ defines the projection onto $\mathfrak{h}$ and $q$ defines the projection onto $\mathfrak{m}$ then since $[\mathfrak{h}, \mathfrak{m}] \subseteq \mathfrak{m}$ for such a space it is obvious that

$$
\sum_{\hookrightarrow} p[q[\xi, \eta], \varsigma]=0
$$

for $\xi, \eta, \varsigma \in \mathfrak{m}$. However, the density manifold $\mathcal{M}$, as a homogeneous space, is not reductive, and the Jacobi identity must be verified directly.

In conclusion, we make a remark on the cotangent bundle $T^{*} \mathcal{M}$ for later reference. It is a theorem that the topological dual of a nonnormable Fréchet space is not itself a Fréchet space. Thus, rather than working with the topological dual of $T \mathcal{M}$, which is a space of distributions, we use the Riemannian structure $\rho \mapsto\left\langle\langle\cdot, \cdot\rangle_{\rho}\right.$ to identify the cotangent bundle geometrically.

To be precise, let b:TM $\rightarrow T^{*} M$ be the bundle isomorphism which is the index lowering action. This induces a bundle isomorphism b:TM $\rightarrow T^{*} \mathcal{M}$ (onto the geometric cotangent bundle) by $X^{b}(\rho)=(X(\rho))^{b}$, and the pairing $\left\langle\langle\cdot, \cdot\rangle_{\rho}\right.$ given by

$$
\left\langle\left\langle X^{\mathrm{b}}, Y\right\rangle\right\rangle_{\rho}(\rho)=\int_{M} X^{\mathrm{b}} \cdot Y(\rho) \rho d_{M} x=\int_{M}\langle X(\rho), Y(\rho)\rangle \rho d_{M} x
$$

describes the action of $T^{*} \mathcal{M}$ on $T \mathcal{M}$. 
4. Geometry of the density manifold. In this section we investigate the fundamental properties of the density manifold by studying the Riemannian and symplectic structures on $T M$.

4.1 The Riemannian structure. Generally speaking, if $\mathcal{E}$ is a vector bundle over the manifold $\mathcal{N}$ with projection $\pi$, the nullspace $\left.\operatorname{ker} \pi_{*}\right|_{z}, z \in T \mathcal{E}$, is the subspace of vertical tangent vectors and is naturally isomorphic to the fiber $\mathcal{E}_{\pi(z)}$. A connection is a choice of a complementary subspace of horizontal vectors. The horizontal lift of a path in the base space is then defined, which for Fréchet manifolds, however, may not exist in general. The following result determines the Riemannian connection on $T \mathcal{M}$, which may be interpreted as a connection for the bundle $\pi v: \mathcal{V} \rightarrow \mathcal{M}$ constructed in the previous section.

THEOREM 4.1. Let $X, Y$, and $Z$ be vector fields on $\mathcal{M}$. The Riemannian connection, denoted $\tilde{\nabla}$, is given by

$$
\tilde{\nabla}_{X} Y(\rho) \equiv \rho \bar{\nabla}_{X} Y(\rho)+\nabla_{X(\rho)} Y(\rho)
$$

where $\bar{\nabla}$ is the canonical flat connection on $T M$.

PROOF. There is a trivial parallel translation map $\bar{\tau}=\mathrm{id}$ on $T \mathcal{M}$, identifying fibers as Fréchet spaces. Since we assume that $\rho \in \mathcal{M}$ satisfies $\rho>0$ locally, the completions of the fibers under the weak Riemannian structure are unitarily equivalent as Hilbert spaces. If $X \in T \mathcal{M}$ and $Z \in T_{X} T \mathcal{M}$ is represented by the curve $t \mapsto W(t), W(0)=X$ then

$$
\bar{K} Z=\lim _{t \rightarrow 0} \frac{\bar{\tau} W(t)-X}{t}
$$

defines the flat connection $\bar{\nabla}$ on $T \mathcal{M}$ by

$$
\bar{K} \circ T X \circ Y=\bar{\nabla}_{X} Y \text {. }
$$

To see that $\bar{\nabla}$ is, in fact, a flat connection notice that by considering $Z: \mathcal{M} \rightarrow T \mathcal{M}$ as a map between Fréchet spaces we have that

$$
\begin{aligned}
&\left(\bar{\nabla}_{X} \bar{\nabla}_{Y} Z-\bar{\nabla}_{Y} \bar{\nabla}_{X} Z-\bar{\nabla}_{\llbracket X, Y \rrbracket} Z\right)(\rho) \\
&=\bar{\nabla}_{X}\left(d Z_{\rho}(-\nabla \cdot(Y \rho))\right)-\bar{\nabla}_{Y}\left(d Z_{\rho}(-\nabla \cdot(X \rho))\right)-d Z_{\rho}(-\nabla \cdot(\llbracket X, Y \rrbracket \rho)) \\
&=d^{2} Z_{\rho}(-\nabla \cdot(Y \rho),-\nabla \cdot(X \rho))+d Z_{\rho}\left(-\nabla \cdot\left(\left(X \circ Y-\left(\Delta_{\rho} S_{X}\right) Y\right) \rho\right)\right) \\
&-d^{2} Z_{\rho}(-\nabla \cdot(X \rho),-\nabla \cdot(Y \rho))-d Z_{\rho}\left(-\nabla \cdot\left(\left(Y \circ X-\left(\Delta_{\rho} S_{Y}\right) X\right) \rho\right)\right) \\
&-d Z_{\rho}(-\nabla \cdot((X \circ Y-Y \circ X+[X, Y]) \rho)) \\
&= 0
\end{aligned}
$$

as in Theorem 3.13 .

Now, in general, a connection $\nabla$ on a manifold $N$, induces a bundle map

$$
K_{\nabla}: T^{2} N \rightarrow T N
$$

such that the diagram

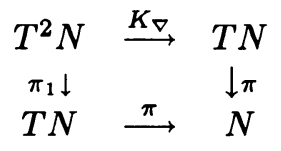


commutes, where $\pi_{1}$ and $\pi$ are the usual projections, and such that $\pi_{1}: T^{2} N \rightarrow T N$ is isomorphic to $\operatorname{ker} K_{\nabla} \oplus \operatorname{ker}\left(\pi_{*}\right)$. Furthermore, $K_{\nabla}$ determines $\nabla$ through the relation

$$
\nabla_{V} W=K \circ T W \circ V .
$$

In this way one constructs the Riemannian connection for the group $D(M)$. Let $K$ be the map associated with the Riemannian connection $\nabla$ on $T M$ as above. If $\hat{K}: T^{2} D(M) \rightarrow T D(M)$ is defined by $\hat{K} Y=K \circ Y$ then the commutativity of the diagram

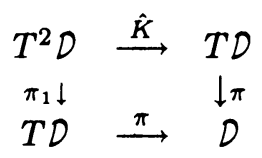

follows from that of the diagram associated with $K$, and the Riemannian connection $\hat{\nabla}$ on $T D(M)$ is then given by

$$
\hat{\nabla}_{X} Y=\hat{K} \circ T Y \circ X
$$

(see [6]). The connection $\tilde{\nabla}$ on $T M$ is then obtained from the fiber bundle structure of $\pi: D(M) \rightarrow \mathcal{M}$ by considering the group action of $D(M)$.

To be concrete, think of $\tilde{\nabla}=\bar{\nabla}+\nabla$ as a sum of the flat connection $\bar{\nabla}$, and the Riemannian connection $\nabla$ on $T M$ acting pointwise on $T \mathcal{M}$. If $f \in C^{\infty}(\mathcal{M})$, notice that

$$
\begin{aligned}
\tilde{\nabla}_{X}(f Y)(\rho) & =\bar{\nabla}_{X}(f Y)(\rho)+\nabla_{X(\rho)}(f(\rho) Y(\rho)) \\
& =X(f)(\rho) Y(\rho)+f(\rho) X \circ Y(\rho)+f(\rho) \nabla_{X(\rho)} Y(\rho) \\
& =\left(X(f) Y+f \tilde{\nabla}_{X} Y\right)(\rho),
\end{aligned}
$$

since $X(\rho) \cdot f(\rho)=0$ when $f(\rho)$ is interpreted as a scalar on $M$ through a sequence

$$
1 \rightarrow C^{\infty}(\mathcal{M}, \mathbf{R})^{*} \rightarrow C^{\infty}\left(\mathcal{M}, C^{\infty}(M, \mathbf{R})\right)^{*} \rightarrow C^{\infty}(M, \mathbf{R})^{*} \rightarrow 1 .
$$

Since linearity is trivial, the Kozul axioms are thus verified. If the curve $t \mapsto \rho(t)$ represents $Z$ at $t=0$, then

$$
\begin{aligned}
Z\left(\langle\langle X, Y\rangle\rangle_{\rho}\right) & =\left.\frac{d}{d t} \int_{M}\langle X(\rho(t)), Y(\rho(t))\rangle \rho(t) d_{M} x\right|_{t=0} \\
& =\int_{M}(\langle Z \circ X, Y\rangle+\langle X, Z \circ Y\rangle) \rho d_{M} x-\int_{M}\langle X, Y\rangle \nabla \cdot(Z \rho) d_{M} x .
\end{aligned}
$$

But the last integral may be written as

$$
\int_{M} Z \cdot\langle X, Y\rangle \rho d_{M} x=\int_{M}\left(\left\langle\nabla_{Z} X, Y\right\rangle+\left\langle X, \nabla_{Z} Y\right\rangle\right) \rho
$$

and thus,

$$
\begin{aligned}
Z\left(\langle\langle X, Y\rangle\rangle_{\rho}\right) & =\left\langle\left\langle Z \circ X+\nabla_{Z} X, Y\right\rangle_{\rho}+\left\langle\left\langle X, Z \circ Y+\nabla_{Z} Y\right\rangle_{\rho}\right.\right. \\
& =\left\langle\left\langle\bar{\nabla}_{Z} X+\nabla_{Z} X, Y\right\rangle\right\rangle_{\rho}+\left\langle\left\langle X, \bar{\nabla}_{Z} Y+\nabla_{Z} Y\right\rangle\right\rangle_{\rho} \\
& =\left\langle\left\langle\tilde{\nabla}_{Z} X, Y\right\rangle_{\rho}+\left\langle\left\langle X, \tilde{\nabla}_{Z} Y\right\rangle\right\rangle_{\rho} .\right.
\end{aligned}
$$


Finally, since

$$
\begin{aligned}
\tilde{\nabla}_{X} Y-\tilde{\nabla}_{Y} X-\llbracket X, Y \rrbracket & \equiv \bar{\nabla}_{X} Y-\bar{\nabla}_{Y} X+\nabla_{X} Y-\nabla_{Y} X+\llbracket X, Y \rrbracket \\
& \equiv X \circ Y-Y \circ X+[X, Y]-\llbracket X, Y \rrbracket \\
& \equiv 0
\end{aligned}
$$

the connection $\tilde{\nabla}$ is clearly torsion-free.

We comment that $\nabla_{X} Y$ is not a gradient vector field, in general, when $X$ and $Y$ are. Thus, the connection $\tilde{\nabla}$ is most naturally viewed as a bundle connection for the bundle $\pi_{\nu}: \mathcal{V} \rightarrow \mathcal{M}$. In so considering $\tilde{\nabla}: \Gamma(\mathcal{V}) \rightarrow \Gamma\left(T^{*} \mathcal{M} \otimes \mathcal{V}\right)$, the covariant derivative $\tilde{\nabla}_{X}$ maps vector fields on $\mathcal{M}$ to equivalence classes of vector fields on $M$. Finally, note that the ring of scalars $C^{\infty}(\mathcal{M})=C^{\infty}(\mathcal{M}, \mathbf{R})$ is canonically embedded in the ring $C^{\infty}\left(\mathcal{M}, C^{\infty}(M, \mathbf{R})\right)$. However, with respect to this larger ring the connection $\tilde{\nabla}$ does not satisfy the Kozul axioms since $\nabla_{X(\rho)}: \Gamma(T M) \rightarrow \Gamma(T M)$ is not linear over $C^{\infty}(M, \mathbf{R})$.

COROLLARY 4.2. Let $t \mapsto \rho(t)$ be a path in $\mathcal{M}$ with $t \mapsto v(t)=v(\rho(t)) \in T_{\rho(t)} \mathcal{M}$ the vector field tangent to $\rho$. Then the path $\rho$ is a geodesic if and only if

$$
\partial v / \partial t+\nabla_{v} v=0 .
$$

PROOF. The expression $\tilde{\nabla}_{v} v \equiv 0$ may clearly be rewritten as (4.5). Notice that there is equality rather than equivalence in (4.5) since from the general identity

$$
L_{u} u^{b}=\left(\nabla_{u} u\right)^{b}+\frac{1}{2} d\langle u, u\rangle
$$

we have that $\left(\nabla_{v} v\right)^{b}=\frac{1}{2} d\langle v, v\rangle$ is exact.

COROLLARY 4.3. $M$ is geodesically incomplete.

Let us now adopt the viewpoint that the curvature of a connection $\nabla$ measures the extent to which the map $X \mapsto \nabla_{X}$ fails to be a Lie algebra homomorphism. Again let $X, Y$, and $Z$ be vector fields on $\mathcal{M}$ and interpret $Z: \mathcal{M} \rightarrow T \mathcal{M}$ as a map between Fréchet spaces. The Riemannian connection $\tilde{\nabla}$ on $T M$ is the sum of a flat connection and the Riemannian connection on $T M$, acting pointwise on $\mathcal{M}$. Thus, we expect the curvature on $T M$ to involve only the pointwise curvature on $T M$, but the projection $Q(\rho)$ onto the gradient part must be accounted for. We are interested in the curvature tensor $\tilde{R}(\omega, X, Y, Z)=\left\langle\langle\omega, \tilde{R}(X, Y) Z\rangle, \omega \in T^{*} \mathcal{M}\right.$, and therefore seek to determine the equivalence class of the algebraic expression

$$
\tilde{R}(X, Y) Z=\tilde{\nabla}_{X} \tilde{\nabla}_{Y} Z-\tilde{\nabla}_{Y} \tilde{\nabla}_{X} Z-\tilde{\nabla}_{\llbracket X, Y \rrbracket} Z
$$

To this end note that

$$
\begin{aligned}
\tilde{\nabla}_{Y} Z(\rho) & \equiv \bar{\nabla}_{Y} Z(\rho)+\nabla_{Y(\rho)} Z(\rho) \\
& \equiv_{\rho} Y \circ Z(\rho)+\nabla_{Y(\rho)} Z(\rho)-P(\rho) \nabla_{Y(\rho)} Z(\rho),
\end{aligned}
$$

where the right side is a gradient vector field. Thus,

(4.7) $\tilde{\nabla}_{X} \tilde{\nabla}_{Y} Z \equiv_{\rho} X \circ\left(Y \circ Z+\nabla_{Y} Z-P \nabla_{Y} Z\right)+\nabla_{X}\left(Y \circ Z+\nabla_{Y} Z-P \nabla_{Y} Z\right)$.

Writing out the expression for $\tilde{\nabla}_{\llbracket X, Y \rrbracket} Z$ gives

$\tilde{\nabla}_{[X, Y]} Z \equiv(X \circ Y-Y \circ X+[X, Y]-P[X, Y]) \circ Z+\nabla_{X \circ Y-Y \circ X+[X, Y]-P[X, Y]} Z$. 
Interchanging the roles of $X$ and $Y$ in (4.7) and forming $\tilde{R}$, we get that

$$
\begin{aligned}
\tilde{R}(X, Y) Z \equiv & X \circ\left(\nabla_{Y} Z-P \nabla_{Y} Z\right)+\nabla_{X}\left(Y \circ Z+\nabla_{Y} Z-P \nabla_{Y} Z\right) \\
& -Y \circ\left(\nabla_{X} Z-P \nabla_{X} Z\right)-\nabla_{Y}\left(X \circ Z+\nabla_{X} Z-P \nabla_{X} Z\right) \\
& -\nabla_{X \circ Y-Y \circ X+[X, Y]-P[X, Y]} Z
\end{aligned}
$$

since $\bar{R}(X, Y) Z=\bar{\nabla}_{X} \bar{\nabla}_{Y} Z-\bar{\nabla}_{Y} \bar{\nabla}_{X} Z-\bar{\nabla}_{\llbracket X, Y \rrbracket} Z \equiv 0$. Consider the term $X \circ \nabla_{Y} Z$. If we express $\nabla_{Y} Z$ in local coordinates on $T M$ then

$$
\left(X \circ \nabla_{Y} Z\right)^{j}=\frac{d}{d t}\left(Y^{i}(\rho(t)) \frac{\partial}{\partial q^{i}} Z^{j}+\Gamma_{i k}^{j} Y^{i}(\rho(t)) Z^{k}(\rho(t))\right),
$$

where $\partial \rho / \partial t=-\nabla \cdot(X \rho)$, and therefore since these operations are smooth,

$$
\begin{aligned}
\left(X \circ \nabla_{Y} Z\right)^{j}= & \left(\frac{d}{d t} Y^{i}(\rho(t))\right) \frac{\partial}{\partial q^{i}} Z^{j}+\Gamma_{i k}^{j}\left(\frac{d}{d t} Y^{i}(\rho(t))\right) Z^{k} \\
& +Y^{i} \frac{\partial}{\partial q^{i}}\left(Z^{j}(\rho(t))\right)+\Gamma_{i k}^{j} Y^{i} \frac{d}{d t} Z^{k}(\rho(t)) \\
= & \left(\nabla_{X \circ Y} Z+\nabla_{Y}(X \circ Z)\right)^{j} .
\end{aligned}
$$

$\tilde{R}$ then simplifies to

$$
\begin{aligned}
\tilde{R}(X, Y) Z \equiv & -X \circ\left(P \nabla_{Y} Z\right)+\nabla_{X} \nabla_{Y} Z-\nabla_{X} P \nabla_{Y} Z+Y \circ\left(P \nabla_{X} Z\right) \\
& -\nabla_{Y} \nabla_{X} Z+\nabla_{Y} P \nabla_{X} Z-\nabla_{[X, Y]} Z+\nabla_{P[X, Y]} Z \\
= & R(X, Y) Z-X \circ\left(P \nabla_{Y} Z\right)+Y \circ\left(P \nabla_{X} Z\right) \\
& -\nabla_{X} P \nabla_{Y} Z+\nabla_{Y} P \nabla_{X} Z+\nabla_{P[X, Y]} Z .
\end{aligned}
$$

Finally, by noticing that

$$
X \circ\left(P \nabla_{Y} Z\right)(\rho) \equiv \frac{\nabla \cdot(X \rho)}{\rho} P \nabla_{Y} Z(\rho)
$$

since $P \nabla_{Y} Z \equiv 0$ we obtain

Proposition 4.4. Let $X, Y$, and $Z$ be vector fields on $\mathcal{M}$ and let $\tilde{R}$ be given by (4.6). Then

$$
\begin{aligned}
\tilde{R}(X, Y) Z \equiv \equiv_{\rho} R(X, Y) Z-\left(\nabla_{X} P \nabla_{Y} Z\right. & -\nabla_{Y} P \nabla_{X} Z-\nabla_{P[X, Y]} Z \\
& \left.+\Delta_{\rho} S_{X} P \nabla_{Y} Z-\Delta_{\rho} S_{Y} P \nabla_{X} Z\right) .
\end{aligned}
$$

In particular, $\mathcal{M}$ has nonzero curvature when the base manifold $M$ is, for example, the $n$-torus $\mathrm{T}^{n}$ provided with the flat metric. In [3], the sectional curvature of the group of volume preserving diffeomorphisms of the torus $\mathrm{T}^{2}$ is described. The expressions even in the low dimensional case are, in fact, quite complicated. We expect that in some sense the curvature of $\mathcal{M}$ must be dual to that of $D_{\mu}(M)$; however, see $\S 5$ for further comment.

4.2 The symplectic structure. When we interpret the cotangent bundle $T^{*} \mathcal{M}$ geometrically using the Riemannian structure $\left\langle\langle\cdot, \cdot\rangle_{\rho}\right.$, as described at the end of $\S 3$, it is clear that the symplectic structure on $T^{*} \mathcal{M}(\approx T \mathcal{M})$ should be

$$
\Omega_{(\rho, v)}\left(\left(v_{1}, z_{1}\right),\left(v_{2}, z_{2}\right)\right)=\left\langle\left\langle z_{1}, v_{2}\right\rangle_{\rho}-\left\langle\left\langle z_{2}, v_{1}\right\rangle_{\rho},\right.\right.
$$

with respect to a local chart. 
In this section we do not interpret $T^{*} \mathcal{M}$ geometrically, but go through the formalism of pulling back the canonical 2-form to $T \mathcal{M}$ using the smooth bundle map $\varphi: T \mathcal{M} \rightarrow T^{*} \mathcal{M}, \varphi(\rho, v)=\left\langle\langle v, \cdot\rangle_{\rho}\right.$. The computations are straightforward. We will work with vectors on $T \mathcal{M}$ and $T^{*} \mathcal{M}$ as ordered pairs without explicitly mentioning local representations as this should cause no confusion.

Let $\pi: T^{*} \mathcal{M} \rightarrow \mathcal{M}$ be the canonical projection, and let $\langle\langle\cdot, \cdot\rangle\rangle$ denote the pairing of $T^{*} \mathcal{M}$ and $T \mathcal{M}$, thinking of $T^{*} \mathcal{M}$ as the functional analytic dual. If $\omega \in T^{*} \mathcal{M}$ and $z \in T_{\omega}\left(T^{*} \mathcal{M}\right)$, then the map

$$
\vartheta_{\omega}: T_{\omega}\left(T^{*} \mathcal{M}\right) \rightarrow \mathbf{R}, \quad \vartheta_{\omega}(z)=\left\langle\left\langle\omega, \pi_{*} z\right\rangle\right\rangle
$$

defines the canonical 1-form on $T^{*} \mathcal{M}$, where $\pi_{*}: T\left(T^{*} \mathcal{M}\right) \rightarrow T \mathcal{M}$ is the induced map of tangent spaces. If $z=((\rho, \omega),(v, \eta))$ then $\pi_{*} z=(\rho, v)$ and $\vartheta_{\omega}(z)=\langle\langle\omega, v\rangle\rangle$.

The canonical 2 -form $\Omega$ is defined as $\Omega=d \vartheta$. Let $z_{1}=\left((\rho, \omega),\left(v_{1}, \omega_{1}\right)\right)$ and $z_{2}=\left((\rho, \omega),\left(v_{2}, \omega_{2}\right)\right) \in T_{\omega}\left(T^{*} \mathcal{M}\right)$. Then from the definition of exterior derivative

$$
\Omega_{\omega}\left(z_{1}, z_{2}\right)=d \vartheta_{\omega}\left(z_{1}, z_{2}\right)=z_{1} \cdot \vartheta_{\omega}\left(z_{2}\right)-z_{2} \cdot \vartheta_{\omega}\left(z_{1}\right)-\vartheta_{\omega}\left(\llbracket z_{1}, z_{2} \rrbracket\right) .
$$

This may be more explicitly expressed as

$$
\begin{aligned}
\Omega_{\omega}\left(z_{1}, z_{2}\right)= & \left(v_{1}, \omega_{1}\right) \cdot\left\langle\left\langle\omega, v_{2}\right\rangle\right\rangle-\left(v_{2}, \omega_{2}\right) \cdot\left\langle\left\langle\omega, v_{1}\right\rangle\right\rangle-\left\langle\left\langle\omega, \llbracket v_{1}, v_{2} \rrbracket\right\rangle\right\rangle \\
= & \left\langle\left\langle\omega, v_{1} \circ v_{2}\right\rangle\right\rangle+\left\langle\left\langle\omega_{1}, v_{2}\right\rangle\right\rangle-\left\langle\left\langle\omega, v_{2} \circ v_{1}\right\rangle\right\rangle \\
& -\left\langle\left\langle\omega_{2}, v_{1}\right\rangle\right\rangle-\left\langle\left\langle\omega, v_{1} \circ v_{2}-v_{2} \circ v_{1}+\left[v_{1}, v_{2}\right]\right\rangle\right\rangle \\
= & \left\langle\left\langle\omega_{1}, v_{2}\right\rangle\right\rangle-\left\langle\left\langle\omega_{2}, v_{1}\right\rangle\right\rangle-\left\langle\left\langle\omega,\left[v_{1}, v_{2}\right]\right\rangle\right\rangle .
\end{aligned}
$$

The familiar (local) formula for the canonical symplectic form is $\Omega_{\omega}\left(z_{1}, z_{2}\right)=$ $\left\langle\omega_{1}, v_{2}\right\rangle-\left\langle\omega_{2}, v_{1}\right\rangle$. Thus, we obtain a correction term involving the pointwise Lie product of vector fields on $M$.

Now, let $\tilde{\Omega}=\varphi^{*} \Omega$ be the pullback form. Since the bundle map $\varphi: T \mathcal{M} \rightarrow T^{*} \mathcal{M}$ is not an isomorphism, $\tilde{\Omega}$ is a weak symplectic form. That is, it is a closed and weakly nondegenerate 2 -form on the double tangent bundle $T^{2} \mathcal{M}=T(T M)$. If $X_{1}, X_{2} \in T^{2} \mathcal{M}$,

$$
\tilde{\Omega}\left(X_{1}, X_{2}\right)=\Omega\left(T \varphi \cdot X_{1}, T \varphi \cdot X_{2}\right),
$$

where $T \varphi_{(\rho, v)}(y, w)=\left(y, D_{\rho}\left\langle\langle v, \cdot\rangle_{\rho} \cdot y+\left\langle\langle w, \cdot\rangle_{\rho}\right)\right.\right.$, with $D_{\rho}$ denoting the derivative with respect to the metric $\left\langle\langle\cdot, \cdot\rangle_{\rho}\right.$. To obtain $\tilde{\Omega}$ explicitly, let

$$
\begin{aligned}
& X_{1}=\left((\rho, v),\left(v_{1}, v_{2}\right)\right) \in T_{(\rho, v)}(T \mathcal{M}), \\
& X_{2}=\left((\rho, v),\left(v_{3}, v_{4}\right)\right) \in T_{(\rho, v)}(T \mathcal{M}) .
\end{aligned}
$$

Then

$$
\begin{aligned}
& \tilde{\Omega}_{(\rho, v)}\left(X_{1}, X_{2}\right)=\tilde{\Omega}_{(\rho, v)}\left(\left(v_{1}, v_{2}\right),\left(v_{3}, v_{4}\right)\right)=\Omega_{\langle\langle v \cdot\rangle\rangle_{\rho}}\left(T \varphi \cdot X_{1}, T \varphi \cdot X_{2}\right) \\
& =\Omega_{\left\langle\langle\cdot\rangle_{\rho}\right.}\left(\left(v_{1}, D_{\rho}\left\langle\langle v, \cdot\rangle_{\rho} \cdot v_{1}+\left\langle\left\langle v_{2}, \cdot\right\rangle_{\rho}\right),\left(v_{3}, D_{\rho}\left\langle\langle v, \cdot\rangle_{\rho} \cdot v_{3}+\left\langle\left\langle v_{4}, \cdot\right\rangle_{\rho}\right)\right)\right.\right.\right.\right.
\end{aligned}
$$

and using the above expression for $\Omega$ this becomes

$$
\begin{aligned}
\tilde{\Omega}_{(\rho, v)}\left(X_{1}, X_{2}\right)= & D_{\rho}\left\langle\left\langle v, v_{3}\right\rangle_{\rho} \cdot v_{1}+\left\langle\left\langle v_{2}, v_{3}\right\rangle_{\rho}\right.\right. \\
& -D_{\rho}\left\langle\left\langle v, v_{1}\right\rangle_{\rho} \cdot v_{3}-\left\langle\left\langle v_{4}, v_{1}\right\rangle_{\rho}-\left\langle\left\langle v,\left[v_{1}, v_{3}\right]\right\rangle\right\rangle_{\rho} .\right.\right.
\end{aligned}
$$

Now,

$$
D_{\rho}\left\langle\left\langle v, v_{3}\right\rangle\right\rangle_{\rho} \cdot v_{1}=-\int_{M}\left\langle v, v_{3}\right\rangle \nabla \cdot\left(v_{1} \rho\right)=\left\langle\left\langle\nabla_{v_{1}} v, v_{3}\right\rangle_{\rho}+\left\langle\left\langle v, \nabla_{v_{1}} v_{3}\right\rangle_{\rho}\right.\right.
$$


so it is a simple matter to check that $\tilde{\Omega}$, in fact, takes the form

$$
\tilde{\Omega}_{(\rho, v)}\left(X_{1}, X_{2}\right)=\tilde{\Omega}_{(\rho, v)}\left(\left(v_{1}, v_{2}\right),\left(v_{3}, v_{4}\right)\right)=\left\langle\left\langle v_{2}, v_{3}\right\rangle_{\rho}-\left\langle\left\langle v_{4}, v_{1}\right\rangle\right\rangle_{\rho},\right.
$$

by using the fact that the connection $\nabla$ is torsion-free and that the $v_{i}$ 's are gradient vector fields. If $q_{1}, \ldots, q_{n}, \dot{q}_{1}, \ldots, \dot{q}_{n}$ are local coordinates on $T M$ and $\tilde{\omega}$ is the 2form $\tilde{\omega}=g_{i j} d \dot{q}^{j} \wedge d q^{i}$, then we may write

$$
\tilde{\Omega}=\int_{M} \tilde{\omega} \rho
$$

By a second order differential equation on $\mathcal{M}$ we mean a section $\xi$ of the double tangent bundle $T(T \mathcal{M})$ such that if $\pi: T \mathcal{M} \rightarrow \mathcal{M}$ is the canonical projection, $\pi_{*} \xi(v)=v$. Locally, this means that the second and third components are equal:

$$
\xi(\rho, v)=((\rho, v),(v, z)), \quad \pi_{*} \xi(\rho, v)=(\rho, v) .
$$

A second order differential equation $\xi$ is called a spray in case for $s \in \mathbf{R}, \xi(s v)=$ $s_{*} s \xi(v)$, where $s$ is identified with the map $s: T \mathcal{M} \rightarrow T \mathcal{M}$ which is scalar multiplication.

Now let $K: T \mathcal{M} \rightarrow \mathbf{R}$ be the kinetic energy map $K(\rho, v)=\frac{1}{2}\|v\|_{\rho}^{2}=\frac{1}{2}\left\langle\langle v, v\rangle_{\rho}\right.$. Let us seek the Hamiltonian vector field associated with $K$; that is, the vector field $S(\rho, v)=\left((\rho, v),\left(f_{1}(\rho, v), f_{2}(\rho, v)\right)\right)$ such that $i_{S} \tilde{\Omega}=-d K$. Computing for $y, w \in T^{2} \mathcal{M}$ yields

$$
d K(\rho, v)(y, w)=-\frac{1}{2} \int_{M}\langle v, v\rangle \nabla \cdot(y \rho)+\int_{M}\langle v, w\rangle \rho=\left\langle\left\langle\nabla_{y} v, v\right\rangle_{\rho}+\left\langle\langle v, w\rangle_{\rho} .\right.\right.
$$

We obtain then the relation

$$
\left\langle\left\langle f_{2}, y\right\rangle_{\rho}-\left\langle\left\langle w, f_{1}\right\rangle_{\rho}=-\left\langle\left\langle\nabla_{y} v, v\right\rangle\right\rangle_{\rho}-\left\langle\langle v, w\rangle_{\rho} .\right.\right.\right.
$$

It follows that $f_{1}(\rho, v)=v$ and $f_{2}(\rho, v)=-\nabla_{v} v$ since $\left\langle\nabla_{y} v, v\right\rangle=\left\langle\nabla_{v} v, y\right\rangle$, and it is also clear that $S$ is, in fact, a spray. We thereby recover the geodesic equations (4.5). A simple computation now gives the expression

$$
\partial^{2} \rho / \partial t^{2}=\nabla \cdot\left(\left(\nabla_{v} v+\left(\Delta_{\rho} S_{v}\right) v\right) \rho\right)
$$

along an integral curve of the geodesic spray.

4.3 A variational principle.

DEFINITION 4.5. Let $\rho_{0}, \rho_{1} \in \mathcal{M}$ be two (not necessarily distinct) densities. The path space $\Omega_{\rho_{0}, \rho_{1}} \mathcal{M}$, or briefly $\Omega \mathcal{M}$, consists of all smooth paths $\rho: I=[0,1] \rightarrow \mathcal{M}$ from $\rho_{0}$ to $\rho_{1}$ in $\mathcal{M}$.

We will not give $\Omega \mathcal{M}$ a topological structure here. (Note that $\Omega \mathcal{M}$ is twice removed from the finite dimensional case.) The tangent space $T_{\rho} \Omega$ at a path $\rho$ will be the vector space of all smooth vector fields $W$ (on $\mathcal{M}$ ) along $\rho$ for which $W(0)=W(1)=0$.

Suppose that $\mathcal{F}: \Omega \mathcal{M} \rightarrow \mathbf{R}$ is a functional on $\Omega \mathcal{M}$. In order to define the induced map of tangent spaces $\mathcal{F}_{*}: T_{\rho} \Omega \rightarrow T_{\mathcal{F}[\rho]} \mathbf{R}$ we make the following standard definition.

DEFINITION 4.6. If $\rho \in \Omega$, a variation of $\rho$ (keeping endpoints fixed) is a smooth function $\bar{\rho}$ defined on $(-\varepsilon, \varepsilon) \times M \times I$, for some $\varepsilon>0$, such that $\bar{\rho}(0, \cdot, t)=\rho(t)$ and $\bar{\rho}(\alpha, \cdot, \cdot) \in \Omega_{\rho_{0}, \rho_{1}} \mathcal{M}$ for each $\alpha \in(-\varepsilon, \varepsilon)$. The variation vector field associated with the variation $\bar{\rho}$ is defined to be the vector field $W \in T_{\rho} \Omega$ given by

$$
W(t)=\left.\frac{\partial}{\partial \alpha} \bar{\rho}(\alpha, \cdot, t)\right|_{\alpha=0} \equiv D_{\alpha} \bar{\rho}(0, \cdot, t) .
$$


Clearly the map $\bar{\rho} \mapsto W \in T_{\rho} \Omega$ is surjective. Thus, if $\mathcal{F}$ is a functional on $\Omega \mathcal{M}$ we may define $\mathcal{F}_{*}: T_{\rho} \Omega \rightarrow T_{\mathcal{F}[\rho]} \mathrm{R}$ by

$$
\mathcal{F}_{*}(W)=d \mathcal{F}[\bar{\rho}(\alpha)] /\left.d \alpha\right|_{\alpha=0}
$$

where $\bar{\rho}$ is a variation of $\rho$ with variation vector field $W$.

DEFINITION 4.7. The path $\rho \in \Omega \mathcal{M}$ is critical for the functional $₹: \Omega \rightarrow \mathbf{R}$ in case

for each variation $\bar{\rho}$ of $\rho$.

$$
\mathcal{F}_{*}\left(D_{\alpha} \bar{\rho}\right)=\left.\frac{d}{d \alpha} \mathcal{F}[\bar{\rho}(\alpha)]\right|_{\alpha=0}=0
$$

So, for example, if $\mathcal{F}$ takes its minimum at $\rho \in \Omega \mathcal{M}$, and if $d \mathcal{F}[\bar{\rho}] / d \alpha$ is always defined, then $\rho$ is a critical path.

The variational principle discussed above provides a convenient and flexible notion of criticality for the density manifold. It is a simple exercise, for example, to verify the geodesic equations by this method.

5. Configuration space quantization. Recall now from Theorem 2.3 that the renormalized classical action associated with a Markovian diffusion $\xi$ having current velocity vector field $v=\frac{1}{2}\left(b+b_{*}\right)$ and osmotic velocity vector field $u=$ $\frac{1}{2}\left(b-b_{*}\right)=\frac{1}{2}(\nabla \rho / \rho)$ is

$$
\begin{aligned}
E\left[\frac{1}{2}\langle b, b\rangle+\frac{1}{2} \nabla \cdot b-\varphi+\frac{1}{12} \bar{R}\right] & =E\left[\frac{1}{2}\langle v, v\rangle-\frac{1}{2}\langle u, u\rangle-\varphi+\frac{1}{12} \bar{R}\right] \\
& =\int_{M}\left(\frac{1}{2}\langle v, v\rangle-\frac{1}{2}\langle u, u\rangle-\varphi+\frac{1}{12} \bar{R}\right) \rho \\
& =\frac{1}{2}\left\langle\langle v, v\rangle_{\rho}-\frac{1}{2}\left\langle\langle u, u\rangle_{\rho}-\int_{M}\left(\varphi-\frac{1}{12} \bar{R}\right) \rho .\right.\right.
\end{aligned}
$$

However, $\left\langle\langle u, u\rangle_{\rho} \in C^{\infty}(\mathcal{M})\right.$ is defined pointwise on $\mathcal{M}$ since $u$ is not a tangent vector. We therefore consider $\mathcal{V}(\rho) \in C^{\infty}(\mathcal{M})$ defined by

$$
\mathcal{V}(\rho)=\frac{1}{2} \int_{M}\langle u, u\rangle \rho+\int_{M}\left(\varphi-\frac{1}{12} \bar{R}\right) \rho
$$

as a scalar potential on $\mathcal{M}$. Now consider a smooth time-dependent 1-form $A \in$ $T^{*} M$, and form the potential

$$
\nu_{\varphi, A}(\rho, v)=\mathcal{V}(\rho)+\int_{M}\left(\frac{1}{2}\left\langle A, A^{\sharp}\right\rangle-\langle A, v\rangle\right) \rho .
$$

While $\mathcal{V}$ is lifted to $T \mathcal{M}$ from $\mathcal{M}, \mathcal{V}_{\varphi, A}$ lives on $T \mathcal{M}$. Using the Riemannian and symplectic structures of $T \mathcal{M}$ we now prove the following.

THEOREM 5.1. The path $\rho \in \Omega \mathcal{M}$ is critical for the action

$$
I_{\varphi, A}[\rho]=\int_{I}\left(\langle v, v\rangle_{\rho}-\nu_{\varphi, A}(\rho, v)\right)
$$

if and only if the Schrödinger equation

$$
i \frac{\partial \psi}{\partial t}=\frac{1}{2}\left(\frac{1}{i} \nabla^{j}-A^{j}\right)\left(\frac{1}{i} \nabla_{j}-A_{j}\right) \psi+\varphi \psi
$$


holds for $\psi=\exp \left(R+i S_{v}+i S_{A}\right)$, where $v=\nabla S_{v}$ is the vector field tangent to $\rho, u=\nabla R=\nabla \log \rho$, and $Q(\rho) A^{\sharp}=\nabla S_{A}$.

PROOF. It follows from general principles that we need to determine the second order vector field $Z \in T^{2} \mathcal{M}$ associated with the pullback form

$$
\Omega_{L_{\varphi, A}}=\left(F L_{\varphi, A}\right)^{*} \Omega
$$

through the relation

$$
i_{Z} \Omega_{L_{\varphi}, A}=d E_{\varphi, A} .
$$

Here $F L: T N \rightarrow T^{*} N$ denotes the fiber derivative of the Lagrangian $L$ and $E_{\varphi, A}$ is the associated energy.

Let us first suppose that $A \equiv 0$. Then since the Lagrangian splits we need only determine the gradient of $\mathcal{V}$ under the weak Riemannian structure $\left\langle\langle\cdot, \cdot\rangle_{\rho}\right.$ on $T \mathcal{M}$. In this case, along an integral curve $t \mapsto(\rho(t), v(t))$ of $Z$ we have that

$$
d v / d t=S(x(t), v(t))-[\operatorname{grad} \mathcal{V}(x(t))]_{v(t)}^{l},
$$

where $[w]_{v}^{l}$ denotes the vertical lift of $w$ to $T_{v}(T \mathcal{M})$, and $S$ is the geodesic spray constructed in the previous section. Some elementary computations show that

$$
d \mathcal{V}(\rho) \cdot v=\left\langle\left\langle v,-\frac{1}{2} \nabla \nabla \cdot u\right\rangle_{\rho}+\left\langle\langle v, \nabla \varphi\rangle_{\rho}-\left\langle\left\langle u, \nabla_{v} u\right\rangle\right\rangle_{\rho} .\right.\right.
$$

But this reduces to

$$
d \mathcal{V}(\rho) \cdot v=\left\langle\left\langle v, \nabla \varphi-\frac{1}{2} \nabla \nabla \cdot u-\nabla_{u} u\right\rangle_{\rho},\right.
$$

since $u$ is a gradient, and therefore

$$
\operatorname{grad} \mathcal{V}(\rho) \equiv_{\rho}-\nabla_{u} u(\rho)-\nabla \nabla \cdot u(\rho)+\nabla \varphi(\rho)
$$

where $\nabla \varphi$ is the constant vector field $\rho \mapsto \nabla \varphi(\rho)=\nabla \varphi$ on $\mathcal{M}$.

Next notice that $\Delta_{\mathrm{DG}}$ commutes with $\nabla$. and $\nabla$. For if $f \in C^{\infty}(M)$ then by Ricci's identity

$$
\nabla_{i} \nabla_{j} \nabla_{k} f=\nabla_{j} \nabla_{i} \nabla_{k} f-R_{k i j}^{l} \nabla_{l} f=\nabla_{j} \nabla_{k} \nabla_{i} f-R_{k i j}^{l} \nabla_{l} f
$$

so that

$$
\nabla_{i} \Delta f=\nabla_{i} \Delta_{\mathrm{DG}} f=\nabla^{k} \nabla_{k} \nabla_{i} f+R_{i}^{k} \nabla_{k} f=\Delta_{\mathrm{DG}} \nabla_{i} f .
$$

We thus have the relation

$$
\partial v / \partial t+\nabla_{v} v-\nabla_{u} u+\frac{1}{2} \Delta_{\mathrm{DG}} u+\nabla \varphi=0
$$

along an integral curve of $Z$.

Let us now consider the 1-form $A$. There are two approaches to obtaining the symplectic gradient associated with the pullback form. The first is to let $L_{A}: T \mathcal{M} \rightarrow$ $\mathbf{R}$ be given by $L_{A}(v)=\left\langle\langle A, v\rangle_{\rho}, v \in T_{\rho} \mathcal{M}\right.$. Then we may form the fiber derivative $F L_{A}: T \mathcal{M} \rightarrow T^{*} \mathcal{M}$,

$$
F L_{A}(v) \cdot w=\left\langle\langle A, w\rangle_{\rho}\right.
$$

and the pullback form $\tilde{\Omega}_{L_{A}}=\left(F L_{A}\right)^{*} \Omega$. If $X_{1}, X_{2} \in T^{2} T \mathcal{M}$ are given by

$$
X_{1}=\left((\rho, v),\left(v_{1}, v_{2}\right)\right), \quad X_{2}=\left((\rho, v),\left(v_{3}, v_{4}\right)\right)
$$


with respect to a local chart, then clearly

$$
\begin{aligned}
\tilde{\Omega}_{L_{A}}\left(X_{1}, X_{2}\right) & =\Omega\left(T F L_{A} \cdot X_{1}, T F L_{A} \cdot X_{2}\right) \\
& =\Omega_{\left\langle\langle A, \cdot\rangle_{\rho}\right.}\left(\left(v_{1}, D_{\rho}\left\langle\langle A, \cdot\rangle_{\rho} \cdot v_{1}\right),\left(v_{3}, D_{\rho}\left\langle\langle A, \cdot\rangle_{\rho} \cdot v_{3}\right)\right) .\right.\right.
\end{aligned}
$$

The second approach, which we adopt here for simplicity, is to work with the variational principle of $\S 4$, and use the basic properties of the family of Laplacians $\rho \mapsto \Delta_{\rho}$. We will then need to note that equation (5.2) takes the equivalent form

$$
\frac{\partial S_{v}}{\partial t}+\frac{1}{2}\langle v, v\rangle+\varphi-\frac{1}{2}\langle u, u\rangle-\frac{1}{2} \nabla \cdot u=0
$$

upon appropriate normalization of the generating function $S_{v}$. To this end let $\bar{\rho}$ be a variation of $\rho$. We need only examine the potential term

$$
I_{A}=\int_{I} \int_{M}\langle A, v\rangle \rho d_{M} x d t
$$

under variations of $\rho$, since it is clear that

$$
D_{\alpha} \int_{M}\left\langle A, A^{\sharp}\right\rangle \bar{\rho}=\int_{M}\left\langle A, A^{\sharp}\right\rangle D_{\alpha} \bar{\rho} \text {. }
$$

For $\rho \in \mathcal{M}$ let $\tilde{A}(\rho) \equiv_{\rho} A(\rho)=A$ be the 1 -form $\tilde{A}(\rho)=\left(Q(\rho) A^{\sharp}\right)^{b}$. Then we have that

$$
I_{A}=\int_{I} \int_{M}\langle\tilde{A}, v\rangle \rho d_{M} x d t
$$

since we assume $v$ to be a gradient. Now, writing $\tilde{A}(\bar{\rho}(\alpha))=\tilde{A}(\alpha)$,

$$
\left.\frac{d I_{A}}{d \alpha}[\bar{\rho}(\alpha)]\right|_{\alpha=0}=\int_{I} \int_{M}\left\langle D_{\alpha} \tilde{A}(\alpha), v\right\rangle \rho d_{M} x d t+\int_{I} \int_{M}\left\langle\tilde{A}, D_{\alpha}(\bar{v} \bar{\rho})\right\rangle d_{M} x d t .
$$

But now $\tilde{A}$ is exact, so a simple manipulation gives

$$
\int_{I} \int_{M}\left\langle\tilde{A}, D_{\alpha}(\bar{v} \bar{\rho})\right\rangle d_{M} x d t=-\int_{I} \int_{M} \frac{\partial S_{A}}{\partial t} D_{\alpha} \bar{\rho} d_{M} x d t
$$

Isolating $D_{\alpha} \bar{\rho}$ in the integral $\int_{I} \int_{M}\left\langle D_{\alpha} \tilde{A}(\alpha), v\right\rangle \rho$ requires the identity

$$
D_{\alpha} \tilde{X}(\bar{\rho}) \equiv_{\rho}(X-Q(\rho) X)\left(D_{\alpha} \bar{\rho} / \rho\right)
$$

for the projection $\tilde{X}=Q X$ of an arbitrary $C^{\infty}$ vector field $X$. To verify this identity let $f \in C^{\infty}\left(\mathcal{M}, C^{\infty}(M)\right)$ be such that $\int_{M} f(\rho) \rho=0$; that is, $f(\rho) \in \mathcal{K}_{\rho}$ for each $\rho \in \mathcal{M}$. Then by smoothness of $\Delta_{\rho}^{-1}$,

$$
D_{\alpha} \Delta_{\bar{\rho}(\alpha)}^{-1} f(\bar{\rho}(\alpha))=\Delta_{\bar{\rho}(\alpha)}^{-1}\left(D_{\alpha} f(\bar{\rho}(\alpha))-\nabla \Delta_{\bar{\rho}(\alpha)}^{-1} f \cdot D_{\alpha}\left(\frac{\nabla \bar{\rho}(\alpha)}{\bar{\rho}(\alpha)}\right)\right)
$$

in particular,

$$
D_{\alpha} f-\nabla \Delta_{\rho}^{-1} f \cdot D_{\alpha}\left(\frac{\nabla \rho}{\rho}\right) \in K_{\rho}=\operatorname{Dom}\left(\Delta_{\rho}^{-1}\right) .
$$

To see this, set $g(\alpha)=\Delta_{\bar{\rho}(\alpha)}^{-1} f(\bar{\rho}(\alpha))$. Then $\Delta_{\bar{\rho}(\alpha)} g(\alpha)=f(\bar{\rho}(\alpha))$ and

$$
D_{\alpha} f=\Delta_{\rho} D_{\alpha} g+D_{\alpha}(\nabla \bar{\rho} / \bar{\rho}) \cdot \nabla g \text {. }
$$


Therefore (5.3) follows by applying $\Delta_{\rho}^{-1}$ to both sides of the equation

$$
\Delta_{\rho} D_{\alpha} g=D_{\alpha} f-D_{\alpha}(\nabla \bar{\rho} / \bar{\rho}) \cdot \nabla \Delta_{\rho}^{-1} f .
$$

Now apply this to the case where $\nabla f(\rho)=Q(\rho) X$. Then

$$
\begin{aligned}
D_{\alpha}(Q(\rho) X) & =\nabla \Delta_{\rho}^{-1}\left(D_{\alpha}\left(\frac{1}{\bar{\rho}} \nabla \cdot(X \bar{\rho})\right)-Q(\rho) X \cdot D_{\alpha}\left(\frac{\nabla \bar{\rho}}{\bar{\rho}}\right)\right) \\
& =\nabla \Delta_{\rho}^{-1}\left(\nabla\left(\frac{D_{\alpha} \bar{\rho}}{\rho}\right) \cdot X-Q(\rho) X \cdot \nabla\left(\frac{D_{\alpha} \bar{\rho}}{\rho}\right)\right) \\
& =\nabla \Delta_{\rho}^{-1}\left(\frac{1}{\rho} \nabla \cdot\left((X-Q(\rho) X) D_{\alpha} \bar{\rho}\right)\right) \\
& \equiv_{\rho}(X-Q(\rho) X) \frac{D_{\alpha} \bar{\rho}}{\rho} .
\end{aligned}
$$

Apply this identity now to the integral $\int_{I} \int_{M}\left\langle D_{\alpha} \tilde{A}(\alpha), v\right\rangle \rho d_{M} x d t$ to obtain

$$
\int_{I} \int_{M}\left\langle D_{\alpha} \tilde{A}(\alpha), v\right\rangle \rho d_{M} x d t=\int_{I} \int_{M}\left\langle A^{\sharp}-Q(\rho) A^{\sharp}, v\right\rangle D_{\alpha} \bar{\rho} d_{M} x d t .
$$

Together with the expression (5.2) it now follows that $\rho \in \Omega \mathcal{M}$ is critical for the Lagrangian $L_{\varphi, A}$ if and only if

$$
\frac{\partial S_{v}}{\partial t}+\frac{1}{2}\langle v, v\rangle+\varphi+\frac{1}{2}\left\langle A, A^{\sharp}\right\rangle+\frac{\partial S_{A}}{\partial t}+\langle\tilde{A}-A, v\rangle-\frac{1}{2}\langle u, u\rangle-\frac{1}{2} \nabla \cdot u
$$

is a function of $t$ alone. By normalization of the generating function $S_{v}+S_{A}$ we may assume that this expression vanishes. Then under the gauge transformation

$$
A \mapsto A-\tilde{A}, \quad \varphi \mapsto \varphi+\frac{\partial S_{A}}{\partial t}
$$

we obtain

$$
\begin{aligned}
\frac{\partial S_{v}}{\partial t} & +\frac{1}{2}\langle v, v\rangle+\varphi+\frac{1}{2}\left\langle A-\tilde{A}, A^{\sharp}-\tilde{A}^{\sharp}\right\rangle+\frac{\partial S_{A}}{\partial t} \\
& +\langle\tilde{A}-A, v\rangle-\frac{1}{2}\langle u, u\rangle-\frac{1}{2} \nabla \cdot u=0,
\end{aligned}
$$

which, together with the continuity equation, is equivalent to the Schrödinger equation with $\psi=\exp \left(R+i\left(S_{v}+S_{A}\right)\right)$, as claimed.

We end this section by commenting briefly on the cases where the densities may have nodes and where the base manifold $M$ is not assumed to be compact.

Let $M$ be a general smooth Riemannian manifold, and let $\mathcal{M}$ be the collection of smooth densities on $M$. If $\rho \in \mathcal{M}$, we may form the osmotic Laplacian $\Delta_{\rho}$ as in $\S 3.3$ : Let $\mathcal{H}^{1}$ be the completion of $C_{c}^{\infty}(M)$, the subspace of $\mathcal{H}=L^{2}\left(d_{M} x\right)$ consisting of all smooth functions which are constant outside a compact set, in the norm associated with the quadratic form

$$
\langle f, g\rangle_{1}=\frac{1}{2} \int_{M}\langle\nabla f, \overline{\nabla g}\rangle_{\rho} d_{M} x+\int_{M} f \bar{g} \rho d_{M} x
$$

Then $\langle\cdot, \cdot\rangle_{1}$ is closeable, and we may let $\frac{1}{2} \Delta_{\rho}$ be the operator corresponding to this quadratic form. 
Now let $t \mapsto \rho(t)$ be a smooth curve in $\mathcal{M}$ with $\rho(0)=\rho$. If the gradient

$$
v=-\nabla \Delta_{\rho}^{-1}\left(\frac{1}{\rho} \frac{\partial \rho}{\partial t}\right)
$$

exists in $L^{2}(\rho)$ then by elliptic regularity for $\Delta_{\rho}$ we may conclude that $v$ is smooth. The definition of variation of compact support is then made in the obvious way, and the above results go through under the appropriate modifications. We recall that for noncompact $M$ the group $D(M)$ becomes less manageable; however, Moser's theorem goes through under suitable restrictions on the densities at infinity [7].

The case where $\rho$ may have nodes is less easily generalized. From the point of view of stochastic mechanics, the nodes of the density are barriers for the diffusion on configuration space. More precisely, the following theorem is proved in [20].

THEOREM 5.2. Suppose that $M$ is compact, and that $\xi$ is a smooth Markovian diffusion satisfying

$$
\frac{1}{2}\left(D D_{*} \xi+D_{*} D \xi\right)=-\nabla \varphi
$$

for some $\varphi \in C^{\infty}(M \times I)$. Let $\rho$ be the density of $\xi$ and set

$$
Z_{\varepsilon}=\{(x, t) \mid \rho(x, t) \leq \varepsilon\} \subseteq M \times I .
$$

Let $\tau_{\varepsilon}$ be the stopping time

$$
\tau_{\varepsilon}=\inf \left\{t \mid(\xi(t), t) \in Z_{\varepsilon}\right\},
$$

with $\tau_{\varepsilon}=\infty$ in the event that $\left\{t \mid(\xi(t), t) \in Z_{\varepsilon}\right\}=\varnothing$ and let $\xi_{\varepsilon}$ be the Markov process defined on $\dot{M}=M \cup\{\infty\}$ by $\xi_{\varepsilon}(s)=\xi\left(s \wedge \tau_{\varepsilon}\right)$ and $\xi_{\varepsilon}\left(\tau_{\varepsilon}\right)=\infty$. If $\operatorname{Pr}_{\varepsilon}$ is the measure on path space corresponding to $\xi_{\varepsilon}$, then

$$
\lim _{\varepsilon \rightarrow 0} \operatorname{Pr}_{\varepsilon}\left(\tau_{\varepsilon}<\infty\right)=0 .
$$

However, for technical reasons, this phenomenon is difficult to investigate in the context of configuration space quantization. Suppose we let $\mathcal{M}_{0}$ be the collection of smooth densities, possibly with nodes, on $M$. As before, we let $\mathcal{N}$ be the Fréchet vector bundle over $M$ consisting of the smooth $n$-forms on $M$, let $K \subseteq \mathcal{N}$ be the closed subbundle

$$
\mathcal{K}=\left\{\eta \in \mathcal{N} \mid \int_{M} \eta=0\right\}
$$

$\overline{\mathcal{K}}$ the affine subspace $\bar{K}+\mu$, and take

$$
\mathcal{M}_{0}=\left\{\rho \in \bar{K} \mid \int_{M} f \rho \geq 0, \forall f \in C_{0,+}^{\infty}(M)\right\}
$$

where $C_{0,+}^{\infty}(M)$ is the collection of smooth nonnegative functions of compact support on $M$. Then $\mathcal{M}_{0}$ is a closed convex subset of a closed affine subspace of $\mathcal{N}$. If $\rho \in \mathcal{M}_{0}$, we will denote by $Z_{\rho}$ the closed subset of $M$ consisting of the zeros of $\rho$; that is, $Z_{\rho}=\{x \in \mathcal{M} \mid \rho(x)=0\}$. Note that the quadratic form construction of the operator $\Delta_{\rho}$ given above applies equally well to the present case where $\rho$ has nodes and $M$ is noncompact. However, suppose that $\rho \in \mathcal{M}_{0}-\mathcal{M}$, and let $t \mapsto \rho(t)$ be a smooth curve in $\mathcal{M}_{0}$ with $\rho(0)=\rho$. Suppose that $\rho_{k} \in \Omega \mathcal{M}$ is a sequence of paths which converge to $\rho$ in the $C^{\infty}$ topology. Then we may try to obtain the tangent to $\rho$ at $t=0$ by considering the operators $\Delta_{\rho_{k}(0)}$. However, the family $\left\{\rho_{k}(0) \Delta_{\rho_{k}(0)}\right\}$ 
is not uniformly elliptic, and thus, it is not clear, in general, how to obtain estimates guaranteeing the existence of $S^{1} \in \mathcal{H}^{1}(\rho)$ which is smooth on $Z_{\rho}^{c}$ and satisfies $\partial \rho / \partial t=-\nabla \cdot(\nabla S \rho)=-\rho \Delta_{\rho} S$. It therefore does not seem possible to give $\mathcal{M}_{0}$ the structure of a Fréchet manifold in a manner analogous to the construction of $\S 3$.

6. The group of diffeomorphisms over the densities. From the functorial relation (1.1) we see that the cotangent map $\mathcal{M} \mapsto T^{*} \mathcal{M}$ yields a mechanical system in $C$ by the procedure of configuration space quantization. In this section we study the fiber bundle $\pi: D(M) \rightarrow \mathcal{M}$ as a means of obtaining a structure theorem relating $C$ and $\mathcal{Q}$.

The essential idea is that the cotangent foliation $T^{*} \mathcal{M} \rightarrow \mathcal{M}$ involves an implicit semidirect product structure since a path of densities is the adjoint flow of the tangent vector fields. To make this structure explicit, we may canonically lift to $T^{*} D(M)$. The group $D_{\mu}(M)$ is then an isotropy subgroup under which the lifted Hamiltonian system is right-invariant. Dividing out by $D_{\mu}(M)$ then leads to a symplectic diffeomorphism with the coadjoint orbits in a semidirect product. The resulting structure is a special case of a general framework studied in [15] and [10]. We shall refer to [15] for many details and further discussion of the general theory.

6.1 The fiber bundle $\pi: D(M) \rightarrow \mathcal{M}$. We begin by collecting some simple facts relating to the principal bundle $\pi: D(M) \stackrel{D_{\mu}(M)}{\longrightarrow} \mathcal{M}$. Recall that projection $\pi$ acts by pushforward of the Riemannian volume element $\mu$, and there is a global trivialization $D(M) \approx D_{\mu}(M) \times \mathcal{M}$. Let $\mathrm{V} \subseteq T D(M)$ denote the vertical bundle. The vertical subspace $\mathrm{V}_{\eta}, \eta \in D(M)$, is given by

$$
\mathrm{V}_{\eta}=\operatorname{ker} T_{\eta} \pi=\left\{X \in T_{\eta} D(M) \mid T_{\eta} \pi(X)=0\right\} \text {. }
$$

Suppose that $X \in \mathrm{V}_{\eta} \subseteq T_{\eta} D(M)$ and let $t \mapsto \eta(t) \in D(M)$ be a curve representing $X$ at $\eta$. Then

$$
T_{\eta} \pi(X)=\left.\frac{d t}{d t}\right|_{t=0}\left(\eta_{t}\right)_{*} \mu=\left.\frac{d}{d t}\right|_{t=0}\left(\eta_{t} \circ \eta^{-1}\right)_{*} \eta_{*} \mu=0
$$

so $\mathrm{V}$ consists of those vector fields $X$ over $\eta$ such that $X \circ \eta^{-1}$ has divergence zero with respect to the pushforward measure $\eta_{*} \mu$.

Let $\mathrm{H}_{\eta}$ be the horizontal subspace

$$
\mathrm{H}_{\eta}=\left\{X \in T_{\eta} D(M) \mid X \circ \eta^{-1}=\nabla S_{X}, S_{X} \in C^{\infty}(M)\right\},
$$

and let $\mathrm{H}=\bigcup_{\eta} \mathrm{H}_{\eta}$ be the corresponding horizontal subbundle. Then clearly

$$
T_{\eta} D(M)=\mathrm{V}_{\eta} \oplus \mathrm{H}_{\eta}, \quad \mathrm{H}_{R_{\xi} \eta}=T R_{\xi} \mathrm{H}
$$

so that $\mathrm{H}$ is a connection with respect to the right action of the structure group $D_{\mu}(M)$ on $D(M)$. (Note that $\mathcal{H}$ is not a connection with respect to the left action.) Moreover, $T D(M)=\mathrm{H} \oplus \mathrm{V}$ is an orthogonal splitting with respect to the weak metric

$$
\langle\langle V, W\rangle\rangle_{\eta}=\int_{M}\langle V(X), W(X)\rangle_{\eta(X)} d_{M} X
$$

Next, let $A \in T_{e} D_{\mu}(M)$ be a divergence-free vector field on $M$. Then $\operatorname{EXP} t A$ represents the flow of $A$, and the fundamental vector field $A^{*}$ is given by

$$
A^{*}(\eta)=\left.\frac{d}{d t} \eta \circ \operatorname{EXP} t A\right|_{t=0}=T \eta \circ A
$$


To verify that $A^{*}(\eta)$ is an element of the vertical subspace $\mathrm{V}_{\eta}$ we simply note that

$$
\begin{aligned}
\left.\frac{d}{d t}\right|_{t=0}(\eta \circ \operatorname{EXP} t A)_{*} \mu & =\left.\frac{d}{d t}\right|_{t=0}\left(\eta \circ \operatorname{EXP} t A \circ \eta^{-1}\right)_{*} \eta_{*} \mu \\
& =L_{T \eta \circ A \circ \eta^{-1}} \eta_{*} \mu=L_{\eta_{*} A} \eta_{*} \mu=\eta_{*} L_{A} \mu=0
\end{aligned}
$$

since the Lie derivative is natural with respect to pushforward. Now define the connection 1-form $\omega$ to be the Lie algebra valued 1-form given by $\omega(X)=A$ in case $A^{*}=\operatorname{vert} X$ where vert $X$ is the vertical projection of $X$. The form $\omega$ is well defined since the group action of $D_{\mu}(M)$ is free and transitive on fibers. We therefore have the identities

$$
\begin{gathered}
\omega(T \eta \circ A)=\omega\left(A^{*}(\eta)\right)=A, \\
\omega(X \circ \eta)=\omega\left(T R_{\eta} \circ X\right)=\operatorname{Ad}_{\eta^{-1}} \omega(X)=\eta^{*} \omega(X),
\end{gathered}
$$

and the curvature form $D \omega$ is defined in the usual way:

$$
D \omega(X, Y)=d \omega(\text { horiz } X, \operatorname{horiz} Y) \text {. }
$$

Suppose we now ask to what extent the geometries of $D_{\mu}(M)$ and $D(M)$ may be used to give information on the geometry of the density manifold $\mathcal{M}$. For the moment let $g_{D_{\mu}(M)}$ and $g_{\mathcal{M}}$ denote the metrics on the manifolds $D_{\mu}(M)$ and $\mathcal{M}$. We may realize the bundle metric $g_{D(M)}$ by setting

$$
g_{\mathcal{D}(M)}(X, Y)=g_{\mathcal{M}}\left(\pi_{*} X, \pi_{*} Y\right)+g_{D_{\mu}(M)}(\omega(X), \omega(Y)) .
$$

Then we have that

$$
\begin{aligned}
g_{\mathcal{D}(M)}\left(T R_{\eta} \circ X, T R_{\eta} \circ Y\right)= & g_{\mathcal{M}}\left(\pi_{*} T R_{\eta} \circ X, \pi_{*} T R_{\eta} \circ Y\right) \\
& +g_{D_{\mu}(M)}\left(\operatorname{Ad}_{\eta^{-1}} \omega(X), \operatorname{Ad}_{\eta^{-1}} \omega(Y)\right) \\
= & g_{\mathcal{M}}\left(\pi_{*} X, \pi_{*} Y\right)+g_{D_{\mu}(M)}\left(\eta^{*} \omega(X), \eta^{*} \omega(Y)\right) .
\end{aligned}
$$

However, the metric on $D_{\mu}(M)$ is not invariant under the adjoint action. Thus, this construction does not yield useful geometrical information. Clearly this is an infinite-dimensional phenomenon. (In fact, a group admits a bi-invariant metric if and only if its image under the adjoint action is relatively compact.) A related observation is simply that $\mathcal{M}$ is not a reductive homogeneous space, in the classical sense, as a result of the relation $\left[T_{\mu} \mathcal{M}, T_{e} D_{\mu}(M)\right] \nsubseteq T_{\mu} \mathcal{M}$. Therefore, the relationship between the geometries of $\mathcal{M}, D(M)$, and $D_{\mu}(M)$ is, indeed, very restricted.

Algebraically, we may set

$$
\Lambda^{k}(\mathfrak{g} / \mathfrak{h})=\left\{\omega \in \Lambda^{k}(\mathfrak{g}) \mid \omega\left(X_{1}, \ldots, X_{k}\right)=0 \text { if some } X_{i} \in \mathfrak{h}\right\}
$$

with $\mathfrak{g}=T_{e} D$ and $\mathfrak{h}=T_{e} D_{\mu}$, and then

$$
\Lambda_{\mu}^{k}(\mathfrak{g} / \mathfrak{h})=\left\{\omega \in \Lambda^{k}(\mathfrak{g} / \mathfrak{h}) \mid \operatorname{Ad}_{D_{\mu}}^{*} \omega=\omega\right\} .
$$

We thereby obtain a mapping $d: \Lambda_{\mu}^{k}(\mathfrak{g} / \mathfrak{h}) \rightarrow \Lambda_{\mu}^{k+1}(\mathfrak{g} / \mathfrak{h})$ given by the exterior derivative, and we infer that the cohomology $H^{k}(\mathcal{M})$ is given by a natural isomorphism

$$
H^{k}(\mathcal{M}) \approx \frac{\operatorname{ker} d: \Lambda_{\mu}^{k}(\mathfrak{g} / \mathfrak{h}) \rightarrow \Lambda_{\mu}^{k+1}(\mathfrak{g} / \mathfrak{h})}{\operatorname{im} d: \Lambda_{\mu}^{k-1}(\mathfrak{g} / \mathfrak{h}) \rightarrow \Lambda_{\mu}^{k}(\mathfrak{g} / \mathfrak{h})}
$$

with the cup product in $H^{*}(\mathcal{M})$ corresponding to the wedge product $\wedge$. However, this isomorphism may not be further reduced using the Riemannian structure. 
6.2 Lifting the Hamiltonian. The choice of a connection on a principal bundle $P \stackrel{K}{\rightarrow} B$ allows one to lift a Hamiltonian on $T^{*} B$ to the cotangent bundle $T^{*} P$ of the total space. Let us now examine explicitly the cotangent lift of the Hamiltonian system of the previous sections. For simplicity, we shall disregard the covector and scalar curvature terms.

To quickly review the standard notations and conventions, let $T \mapsto \eta_{t}$ be a curve of diffeomorphisms. We let $X_{1}, \ldots, X_{n}$ denote a generic coordinate chart on $M$, and $x_{1}, \ldots, x_{n}$ the configuration under the motion of the diffeomorphism group; that is, $x_{t}=x(X, t)=\eta_{t}(X)$. The Lagrangian velocity $V$ is given by $V_{t}(X)=$ $V(X, t)=\partial \eta_{t}(X) / \partial t$, and the Eulerian velocity $v$ is determined by $v_{t} \circ \eta_{t}=V_{t}$. We therefore have a commutative diagram

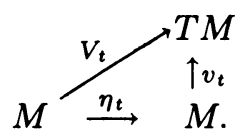

The cotangent bundle $T^{*} D(M)$ consists of 1 -form densities over the diffeomorphisms, so that

$$
T_{\eta}^{*} D(M)=\left\{\alpha_{\eta}: M \rightarrow T^{*} \mathcal{M} \otimes \Lambda^{n}(M) \mid \alpha_{\eta}(X) \in T_{\eta(X)}^{*} M \otimes \Lambda_{X}^{n}(M)\right\}
$$

and the pairing between $T_{\eta} D(M)$ and $T_{\eta}^{*} D(M)$ is given by

$$
\langle\alpha, V\rangle=\int_{M} \alpha(X) \cdot V(X) \text {. }
$$

We therefore take the convention that the bundle metric $\langle\langle\cdot, \cdot\rangle\rangle$ on $T D(M)$ induces the weak metric

$$
\left\langle\langle\alpha, \beta\rangle_{\eta}=\int_{M}\left\langle(\alpha / \mu)^{\sharp},(\beta / \mu)^{\sharp}\right\rangle_{\eta(X)} \rho_{0}(X)\right.
$$

on $T^{*} D(M)$, where $\rho_{0} \in \mathcal{M}$ is a given initial density. In short, the bundle isomorphism $T D(M) \rightarrow T^{*} D(M)$ is described by the index lowering action.

DEFINITION 6.1. Let $\rho_{0} \in \mathcal{M}, \eta \in D(M)$, and let $Q: \mathcal{X}(M) \rightarrow \mathcal{X}(M)$ be the projection operator constructed in $\S 3$. For $\eta \in D(M)$, we define $Q_{\eta}: T_{\eta} D(M) \rightarrow$ $T_{\eta} D(M)$ by

$$
Q_{\eta} V_{\eta}=\left(Q\left(\eta_{*} \rho_{0}\right) V \circ \eta^{-1}\right) \circ \eta=T R_{\eta} \circ Q\left(\eta_{*} \rho_{0}\right) \circ T R_{\eta^{-1}} V .
$$

From the results of $\S 3$ concerning the osmotic Laplacian we have (see also [6])

PROPOSITION 6.2. $Q_{\eta}$ is a smooth operator on $T_{\eta} D(M)$.

To lift the Hamiltonian

$$
\begin{aligned}
H(\rho, v) & =\frac{1}{2} \int_{M}\langle v, v\rangle \rho+\frac{1}{2} \int_{M}\langle u, u\rangle \rho+\int_{M} \varphi \rho \\
& =K(\rho, v)+\mathcal{V}(\rho)
\end{aligned}
$$

to the cotangent bundle $T^{*} D(M)$, we first treat the potential term. Identifying the density $\rho_{0}$ with the function $\rho_{0} / \mu$, the pushforward density $\rho=\eta_{*} \rho_{0}$ is identified with the function $\rho(x)=\rho_{0}(X) J_{\eta}^{-1}(X)$, where $J_{\eta}$ is the Jacobian determinant. Therefore,

$$
\int_{M} \varphi \rho=\int_{M} \varphi(\eta(X)) \rho_{0}(X) d_{M} X .
$$


Similarly, if $T_{X} \eta$ denotes the Jacobian matrix $d x / d X$ then

$$
u(x)=\frac{1}{2} \frac{\nabla_{x} \rho(x)}{\rho(x)}=\frac{1}{2} \frac{\left(T_{X} \eta\right)^{-1} \nabla_{X}\left(\rho_{0}(X) J_{\eta}^{-1}(X)\right)}{\rho_{0}(X) J_{\eta}^{-1}(X)}=U_{\eta}(X)
$$

and thus

$$
\begin{aligned}
\mathcal{V}(\eta) & \equiv \mathcal{V}(\rho)=\mathcal{V}\left(\eta_{*} \rho_{0}\right) \\
& =\frac{1}{2} \int_{M}\left\langle U_{\eta}(X), U_{\eta}(X)\right\rangle \rho_{0}(X) d_{M} X+\int_{M} \varphi(\eta(X)) \rho_{0}(X) d_{M} X,
\end{aligned}
$$

which lives on $D(M)$.

Finally, letting $\alpha \in T^{*} D(M)$ correspond to $V \in T D(M)$ under the isomorphism $T D(M) \rightarrow T^{*} D(M)$ we form

$$
H_{\rho_{0}}\left(\eta, \alpha_{\eta}\right)=\frac{1}{2}\left\langle\left\langle Q_{\eta} V_{\eta}, Q_{\eta} V_{\eta}\right\rangle\right\rangle+\mathcal{V}(\eta) .
$$

It is instructive to check directly that this lift is vertical.

Proposition 6.3. The Hamiltonian $H_{\rho_{0}}$ given above depends smoothly on $\rho_{0}$, and is right invariant under the action of the subgroup $D_{\rho_{0}}(M)=\left\{\eta \in D(M) \mid \eta_{*} \rho_{0}=\right.$ $\left.\rho_{0}\right\}$ of diffeomorphisms which preserve the density $\rho_{0}$.

PROOF. Since the projection $Q(\rho)$ depends smoothly on $\rho$, the Hamiltonian clearly depends smoothly on $\rho_{0}$. Invariance of the potential $\mathcal{V}(\eta)$ under the action of $D_{\rho_{0}}(M)$ is also clear by change of variables.

It remains to check equivariance of the projections $Q_{\eta}$. To this end, let $\phi \in$ $D_{\rho_{0}}(M), \eta \in D(M)$, and $V_{\eta} \in T_{\eta} D(M)$ and observe that

$$
\begin{aligned}
Q_{\eta \circ \phi} T R_{\phi} \circ V_{\eta} & =Q_{\eta \circ \phi} V_{\eta} \circ \phi=\left(Q\left((\eta \circ \phi)_{*} \rho_{0}\right) V_{\eta} \circ \phi \circ(\eta \circ \phi)^{-1}\right) \circ \eta \circ \phi \\
& =\left(Q\left(\eta_{*} \circ \phi_{*} \rho_{0}\right) V_{\eta} \circ \eta^{-1}\right) \circ \eta \circ \phi \\
& =\left(Q\left(\eta_{*} \rho_{0}\right) V_{\eta} \circ \eta^{-1}\right) \circ \eta \circ \phi=T R_{\phi} \circ Q_{\eta} V_{\eta} .
\end{aligned}
$$

Thus the diagram

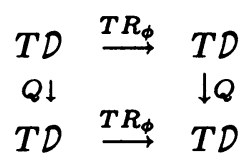

is commutative. In addition, it is a simple matter to check that

$$
\left\langle\left\langle T R_{\phi} V_{\eta}, T R_{\phi} V_{\eta}\right\rangle_{\eta \circ \phi}=\left\langle\left\langle V_{\eta}, V_{\eta}\right\rangle_{\eta} .\right.\right.
$$

Therefore, since the bundle map $T D(M) \rightarrow T^{*} D(M)$ is equivalent to the index lowering action, we now have that $H_{\rho_{0}}$ is $D_{\rho_{0}}(M)$-invariant.

6.3 Reduction and semidirect product structure. The invariance under $D_{\rho_{0}}(M)$ of $H_{\rho_{0}}$ on $T^{*} D(M)$ leads to Lie-Poisson equations on the dual of a semidirect product Lie algebra. The equations are precisely those obtained by the configuration space quantization of the previous section. Our approach here is based on the work of Marsden, Ratiu, and Weinstein [15], and we therefore first summarize the essential points of the theory in the following two theorems.

Let $\gamma: G \rightarrow \operatorname{Aut}(V)$ be a representation of the Lie group $G$ on the topological vector space $V$. The induced Lie algebra homomorphism is denoted $\gamma^{\prime}: \mathfrak{g} \rightarrow \operatorname{End}(V)$. 
Let $S=G \ltimes_{\gamma} V$ be the semidirect product which is the manifold $G \times V$ together with the group law

$$
\left(g_{1}, v_{1}\right)\left(g_{2}, v_{2}\right)=\left(g_{1} g_{2}, v_{1}+\gamma\left(g_{1}\right) v_{2}\right) .
$$

If $f: \mathfrak{g}^{*} \rightarrow \mathbf{R}$ then for $\mu \in \mathfrak{g}^{*}, \delta f / \delta \mu \in \mathfrak{g}$ denotes the functional derivative defined dually by

$$
d f(\mu) \cdot \nu=\langle\nu, \delta f / \delta \mu)
$$

where $d f$ is the Fréchet derivative and $\langle\cdot, \cdot\rangle$ denotes the dual pairing of $\mathfrak{g}^{*}$ and $\mathfrak{g}$. The Lie-Poisson bracket on $\mathfrak{s}^{*}$ is then given by

$$
\{F, G\}(\mu, w)=\left\langle\mu,\left[\frac{\delta F}{\delta \mu}, \frac{\delta G}{\delta \mu}\right]\right\rangle+\left\langle w, \gamma^{\prime}\left(\frac{\delta F}{\delta \mu}\right) \cdot \frac{\delta G}{\delta w}-\gamma^{\prime}\left(\frac{\delta G}{\delta \mu}\right) \cdot \frac{\delta F}{\delta w}\right\rangle
$$

with $F, G: \mathfrak{s}^{*} \rightarrow \mathbf{R}, \delta F / \delta \mu \in \mathfrak{g}$, and $\delta F / \delta W \in V$.

THEOREM 6.4. Let $J$ be the moment map for the right action of the semidirect product $S=G \ltimes_{\gamma} V$ on $T^{*} S$, and let $\tilde{J}: T^{*} G \ltimes V^{*} \rightarrow \mathfrak{s}^{*}$ be given by

$$
\tilde{J}\left(\alpha_{g}, p\right)=\left(T_{e}^{*} R_{g}\left(\alpha_{g}\right), \gamma^{*}\left(g^{-1}\right) p\right),
$$

where $\alpha_{g} \in T_{g}^{*} G$ and $p \in V^{*}$. Then $\tilde{J}$ is a moment map for the action of $G \times_{\gamma} V$ on the Poisson manifold $T^{*} G \times V^{*}$, and both $\tilde{J}$ and $J$ are canonical. Furthermore, there is a canonical map I such that the diagram

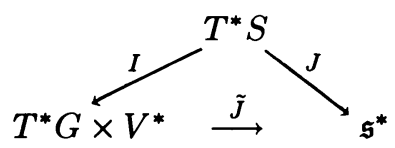

commutes.

A further analysis shows a more detailed picture. In particular, an analysis of the symplectic leaves of $T^{*} G \times V^{*}$ shows that if the given Hamiltonian $H_{p}$ is right invariant under $G_{p}=\left\{g \mid \gamma^{*}(g) p=p\right\}$, then $H_{p}$ induces a Hamiltonian on $T^{*} G / G_{p}$, and via the moment map the reduced space $J^{-1}\left(O_{\mu}\right) / G_{p}$, where $O_{\mu}$ is the coadjoint orbit of $G$ in $\mathfrak{g}^{*}$, is symplectically diffeomorphic to the coadjoint orbit $S \cdot(\mu, p), \mu \in \mathfrak{g}^{*}$, in $\mathfrak{s}^{*}$. Varying $p$ gives a Hamiltonian on $\mathfrak{s}^{*}$, and one is led to derive Lie-Poisson equations on $\mathfrak{s}^{*}$, where the bracket is explicit.

THEOREM 6.5. The family $\left\{H_{p}\right\}_{p \in V^{*}}$ of Hamiltonians induces a Hamiltonian $H$ on $\mathfrak{s}^{*}$ by

$$
H\left(\left(T_{e} R_{g}\right)^{*} \alpha_{g}, \gamma^{*}\left(g^{-1}\right) p\right)=H_{p}\left(\alpha_{g}\right),
$$

with associated Lie-Poisson equations on $\mathfrak{s}^{*}$. In particular, the curve $c_{p}(t) \in T^{*} G$ is a solution of Hamilton's equations for $H_{p}$ if and only if $\tilde{J}\left(c_{p}(t), p\right)$ is a solution of the Hamiltonian system $X_{H}$ on $\mathfrak{s}^{*}$. In addition, the evolution of $p$ is determined by

$$
p(t)=\gamma\left(c_{p}(t)^{-1}\right)^{*} p .
$$

6.4 Semidirect products and the density manifold. Let us now return to the bundle $\pi: D(M) \rightarrow \mathcal{M}$ and recall some basic facts regarding the group action of $D(M)$. Consider the right action $(\eta, \zeta) \mapsto R_{\eta} \zeta=\varsigma \circ \eta$ of $D(M)$ on itself. The cotangent lift 
of this action is described by $\left(\eta, \alpha_{\varsigma}\right) \mapsto\left(T_{\zeta \circ \eta} R_{\eta^{-1}}\right)^{*} \alpha_{\varsigma}$ and the associated moment map is given by

$$
J_{L}\left(\alpha_{\eta}\right)=\left(T_{e} L_{\eta}\right)^{*} \alpha_{\eta}
$$

which is left-inariant. The left action induces the cotangent lift

$$
\left(\eta, \alpha_{\varsigma}\right) \mapsto\left(T_{\eta \circ \varsigma} L_{\eta^{-1}}\right)^{*} \alpha_{\varsigma}
$$

with right-invariant moment map

$$
J_{R}\left(\alpha_{\eta}\right)=\left(T_{e} R_{\eta}\right)^{*} \alpha_{\eta}
$$

where $T_{e} R_{\eta}$ is given by right translation. Thus, $J_{R}\left(\alpha_{\eta}\right) \xi=\left\langle\alpha_{\eta}, \xi \circ \eta\right\rangle$.

The vector space in the appropriate semidirect product should be identified with the densities, which is however not a vector space. We therefore take $V=$ $\mathcal{F}(M)\left(=C^{\infty}(M)\right)$ and identify the dual $\mathcal{F}^{*}(M)$ geometrically with the densities. Let $\gamma: D(M) \rightarrow \operatorname{Aut}(\mathcal{F}(M))$ be the representation given by pushforward. Then the induced Lie algebra representation $\gamma^{\prime}: \mathcal{X}(M) \rightarrow \operatorname{End}(\mathcal{F}(M))$ is minus the Lie derivative, $\gamma^{\prime}(v) f=-L_{v} f$. From the general form of the Lie-Poisson bracket on the dual of a semidirect product Lie algebra we now easily infer that the bracket on $\left(\mathcal{X}(M) \ltimes_{\gamma^{\prime}} C^{\infty}(M)\right)^{*}$ is determined by

$$
\{F, G\}(v, \rho)=\int_{M}\left\langle v,\left[\frac{\delta G}{\delta v}, \frac{\delta F}{\delta v}\right]\right\rangle \rho+\int_{M}\left(L_{\delta G / \delta v} \frac{\delta F}{\delta \rho}-L_{\delta F / \delta v} \frac{\delta G}{\delta \rho}\right) \rho .
$$

We remark that the Poisson structure described above is essentially the structure for compressible flow constructed in [15]. To adapt it to our purposes we must take into consideration the projection operator $Q$.

Under the isomorphism $M \approx \mathcal{F}^{*}(M)$ the induced representation is again pushforward, and for $\rho_{0} \in \mathcal{M}, D_{\rho_{0}}(M)$ is now an isotropy subgroup. The moment map $\tilde{J}$ for the action of the semidirect product $D(M) \ltimes_{\gamma} \mathcal{F}(M)$ on $T^{*} D(M) \times \mathcal{F}^{*}(M) \approx$ $T^{*} D(M) \times M$ is

$$
\tilde{J}\left(\alpha_{\eta}, \rho\right)=\left(\left(T_{e} R_{\eta}\right)^{*} \alpha_{\eta}, \eta^{*} \rho\right) .
$$

Let $H$ be the Hamiltonian on $T^{*} D(M) \times \mathcal{M}$ given by $H\left(\alpha_{\eta}, \rho\right)=H_{\rho}\left(\alpha_{\eta}\right)$, where $H_{\rho}$ is given in $\S 5$. Then by forming the Hamiltonian $\tilde{H}: \mathfrak{s}^{*} \rightarrow \mathbf{R}$ through the composition $\tilde{H} \circ \tilde{J}=H$ we reduce to a Hamiltonian system on the dual of the semidirect product Lie algebra $\mathfrak{s}=\chi(M) \ltimes_{\gamma^{\prime}} \mathcal{F}(M)$.

It is now straightforward to check that the equations of configuration space quantization are obtained by solving for the Lie-Poisson equations $\dot{F}=\{F, H\}$. We omit the details; a similar calculation is carried out in the following section.

Finally, we make the following remark which suggests a more refined structure for the present situation of lifting a Hamiltonian. Let $X$ be a vector field on $M$. Then $\tilde{X}(\eta)=X \circ \eta$ determines a right-invariant vector field on $D(M)$. Now form the vector field $Q_{\eta} \tilde{X}(\eta) \in T_{\eta} D(M)$, which is again right invariant under the induced action of $D(M)$. From the results of $\S 3$, we infer that

$$
\begin{aligned}
& {\left[Q_{\eta} \tilde{X}(\eta), Q_{\eta} \tilde{Y}(\eta)\right](e)} \\
& \quad=Q(\mu)(\nabla \cdot Y(X-Q(\mu) X))-Q(\mu)(\nabla \cdot X(Y-Q(\mu) Y))+[Q(\mu) X, Q(\mu) Y] \\
& \quad=Q_{e}\left(\nabla \cdot Y\left(X-Q_{e} X\right)-\nabla \cdot X\left(Y-Q_{e} Y\right)\right)+\left[Q_{e} X, Q_{e} Y\right]
\end{aligned}
$$


and that the Jacobi identity holds under projection by $Q_{e}$. Since we may replace the form $\mu$ with any density $\rho \in \mathcal{M}$, we obtain a one-parameter family of Lie algebras $\mathcal{Q}_{\rho}(M)$ which does not correspond to any one-parameter family of subgroups of the diffeomorphism group $D(M)$. Since the Hamiltonian on $T^{*} D(M)$ involves the projection onto the cotangent bundle of $T^{*} \mathcal{M}$, in actuality, we are solving for the Lie-Poisson equations in the one-parameter family of duals of semidirect product Lie algebras $\mathfrak{s}_{\rho}=\mathcal{Q}_{\rho} \ltimes_{\gamma^{\prime}} \mathcal{F}(M)$. Thus, lifting to a degenerate Hamiltonian on the total space simply allows the use of the underlying Lie group $D(M)$ to obtain a single semidirect product Lie algebra $\mathfrak{s}$ in which the family $\left\{\mathfrak{s}_{\rho}\right\}$ is embedded.

6.5 A Poisson map $\wp: \mathfrak{s}^{*} \rightarrow \mathcal{H}$. Consider now the symplectic space $(\mathcal{H}, \omega)$, where $\mathcal{H}$ is the complex Hilbert space $\mathcal{H}=L^{2}\left(d_{M} x\right)$ and $\omega$ is the symplectic form $\omega(X, Y)=\operatorname{I} m\langle X, Y\rangle_{\mathcal{H}}$, with $\langle\cdot, \cdot\rangle_{\mathcal{H}}$ the $L^{2}$ inner product of $\mathcal{H}$. Let $\mathcal{F}_{\mathcal{H}}$ denote the collection of maps $f_{H}: \not H \rightarrow \mathbf{R}$ given by

$$
f_{H}(\varphi)=\frac{1}{2}\langle H \varphi, \varphi\rangle_{\mathcal{H}},
$$

where $H$ is a selfadjoint operator on $\mathcal{H}$. The Hamiltonian vector field $X_{f_{H}} \equiv X_{H}$ corresponding to $f_{H}$ is given by

$$
X_{H}(\varphi)=i H \varphi .
$$

The following provides a canonical mapping between $\mathcal{H}$ and $\mathfrak{s}^{*}$.

THEOREM 6.6. Let $\mathfrak{s}^{*}$ be the dual of the semidirect product Lie algebra $\mathcal{X}(M)$ $\ltimes_{\gamma^{\prime}} C^{\infty}(M)$ constructed in the previous section. Let $\wp: \mathfrak{s}^{*} \rightarrow \not$ be given by

$$
\wp(\alpha, \rho)=e^{R+i S}
$$

where $\nabla R=u=\frac{1}{2} \nabla \rho / \rho$, and $\nabla S=Q(\rho) v$ when $v^{b}=\alpha / \mu$. Then $\wp$ is a Poisson map when we restrict to those $f_{H} \in \mathcal{F}_{\mathcal{H}}$ with $H$ of the form $H=-\Delta+\varphi$, for some smooth potential $\varphi: M \rightarrow \mathbf{R}$ on $M$.

As the following proof shows, one may consider more general selfadjoint operators $H=-\Delta+\varphi$ by taking $\varphi$ to be a Rellich class potential, for example.

Proof. Let $H=-\Delta+V$ and $G=-\Delta+U$, where $V$ and $U$ are smooth potentials. We have that

$$
\left\{f_{H}, f_{G}\right\}_{\mathcal{H}}(\psi)=\omega\left(X_{H}, X_{G}\right)(\psi)=\mathfrak{I} m\langle i H \psi, i G \psi\rangle_{\mathcal{H}} .
$$

If we let $\psi=e^{R+i S}$ then a simple computation shows that this reduces to

$$
\left\{f_{H}, f_{G}\right\}_{\mathcal{H}}(\psi)=\int_{M}(V-U)(\Delta S+2\langle u, \nabla S\rangle) \rho .
$$

Another computation shows that

$$
f_{H} \circ \wp(\alpha, \rho)=\frac{1}{2} \int_{M}(\langle Q(\rho) v, Q(\rho) v\rangle-\nabla \cdot u-\langle u, u\rangle+V) \rho
$$

with $(\alpha / \mu)=v^{b}$.

Now, thinking of $f_{H} \circ \wp$ and $f_{G} \circ \wp$ as functions of $\rho$ and $v$ (via the index lowering action) we see that

$$
\frac{\delta f_{G} \circ \wp}{\delta v}(v, \rho)=\frac{\delta f_{H} \circ \wp}{\delta v}(v, \rho)=Q(\rho) v \in \chi(M) .
$$


Furthermore,

$$
\frac{\delta}{\delta \rho}\left(\int_{M} U \rho\right)=U \in C^{\infty}(M)
$$

and

$$
\frac{\delta}{\delta \rho}\left(\int_{M} V \rho\right)=V \in C^{\infty}(M)
$$

We therefore have that

$$
\begin{aligned}
\left\{f_{H} \circ \wp, f_{G} \circ \wp\right\}(v, p)= & -\int_{M}\left\langle v,\left[\frac{\delta f_{H} \circ \wp}{\delta v}, \frac{\delta f_{G} \circ \wp}{\delta v}\right]\right\rangle \rho \\
& +\int_{M}\left(L_{\delta f_{H} \circ \wp / \delta v}\left(\frac{\delta f_{G} \circ \wp}{\delta \rho}\right)-L_{\delta f_{G} \circ \wp / \delta v}\left(\frac{\delta f_{H} \circ \wp}{\delta \rho}\right)\right) \rho \\
= & -\int_{M}\langle v,[Q(\rho) v, Q(\rho) v]\rangle \rho+\int_{M}\left(L_{Q(\rho) v} U-L_{Q(\rho) v} V\right) \rho \\
= & \int_{M}\left(L_{Q(\rho) v} U-L_{Q(\rho) v} V\right) \rho
\end{aligned}
$$

since the terms containing

$$
\frac{\delta}{\delta \rho} \int_{M}(\nabla \cdot u+\langle u, u\rangle) \rho
$$

are common to both $\delta f_{G \circ \wp} / \delta \rho$ and $\delta f_{H \circ \wp} / \delta \rho$ and therefore cancel. Simplifying, we are left with

$$
\begin{aligned}
\left\{f_{H} \circ \wp, f_{G} \circ \wp\right\}(v, p) & =\int_{M}(\nabla S \cdot \nabla U-\nabla S \cdot \nabla V) \rho \\
& =\int_{M}(V-U)(\Delta S+2\langle u, \nabla S\rangle) \rho
\end{aligned}
$$

and the result follows.

It is now a simple matter to check that the multiplicative action of $S^{1}$ on the collection of unit vectors of $\not$ is symplectic, with moment map

$$
\left\langle J_{\mathcal{H}}(\psi), x\right\rangle=(x / 2)\|\psi\|^{2} .
$$

The reduced space is the projective Hilbert space $\hat{H}$, which is the symplectic space of rays in $\mathscr{H}$, and the mapping $\wp$ pulls the action of $S^{1}$ on $\mathscr{H}$ back to the identity on $\mathfrak{s}^{*}$. To summarize, we have the diagram

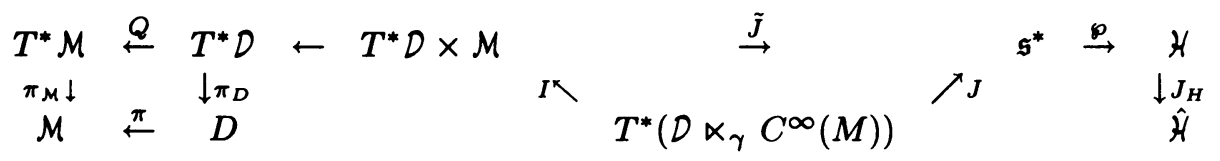

In conclusion we comment on the indeterminancy in the generating function $S_{v}$ by considering the manifold of $N$ indistinguishable particles in $M$, denoted $B_{N}(M)$. To be precise, let $n=\operatorname{dim}(M) \geq 2$, set

$$
F_{N}(M)=\left\{\left(x_{1}, \ldots, x_{N}\right) \in \prod_{i=1}^{N} M \mid x_{i} \neq x_{j} \text { if } i \neq j\right\},
$$

and form an equivalence relation on $F_{N}(M)$ by setting $x \sim x^{\prime}$ in case the coordinates of $x$ differ from the coordinates of $x^{\prime}$ by an element of $S_{N}$, the symmetric group on 
$N$ letters. The manifold $B_{N}(M)$ is obtained from $F_{N}(M)$ modulo this equivalence relation. The fundamental group $\pi_{1} F_{N}(M)$ of $F_{N}(M)$ is the pure braid group with $N$ strings, and the fundamental group of $\pi_{1} B_{N}(M)$ is called the (full) braid group of $M$. It is clear then that the natural projection

$$
\rho: F_{N}(M) \rightarrow B_{N}(M)
$$

is a covering map, with group of covering transformations $S_{N}$. Therefore there is a canonical isomorphism $\pi_{1} B_{N}(M) / \pi_{1} F_{N}(M) \approx S_{N}$. If we choose a base point $x_{0} \in F_{N}(M)$ for $\pi_{1} F_{N}(M)$ and let $\tilde{x}_{0} \in B_{N}(M)$ be such that $\rho\left(x_{0}\right)=\tilde{x}_{0}$, then any element of $\pi_{1} B_{N}(M)$ represented by a loop

$$
\tilde{\gamma}: I \rightarrow B_{N}(M), \quad \tilde{\gamma}(0)=\tilde{\gamma}(1)=\tilde{x}_{0},
$$

lifts uniquely to a path $\gamma: I \rightarrow F_{N}(M)$ with $\gamma(0)=x_{0}$, and $\gamma(0) \mapsto \gamma(1)$ defines a homomorphism $\sigma: \pi_{1}\left(B_{N}(M), \tilde{x}_{0}\right) \rightarrow S_{N}$, with $\operatorname{ker} \sigma=\pi_{1} F_{N}(M)$. In the case that $\operatorname{dim}(M) \geq 3$ and $\pi_{1}(M)=0$, then $\pi_{1} F_{N}(M)=0$ and $F_{N}(M)$ is the universal covering space of $B_{N}(M)$ (essentially since any knot may be untied in four dịmensions).

Now consider a critical path of densities on $B_{N}(M)$, with tangent $v=\nabla S_{v}$. If $\gamma$ is a loop in $B_{N}(M)$, it may be that tracing the value of $S_{v}$ around $\gamma$ results in initial and final values differing by an additive constant. If we require that $\psi=e^{R+i S_{v}}$ be smooth across the nodes of density, then $\psi$ changes by a multiplicative constant $\zeta(\gamma)$ of modulus 1 which only depends on the homotopy class of $\gamma$ in $\pi_{1} B_{N}(M)$. Since $\zeta: \pi_{1} B_{N}(M) \rightarrow \mathrm{C}$ is a homomorphism, by restricting to $\pi_{1} B_{N}(M) / \pi_{1} F_{N}(M)$ we obtain a character of $S_{N}$. If $\pi_{1} F_{N}(M)=0$ then $\psi$ lifts to a well-defined wave function on $F_{N}(M)$, which is either a symmetric or antisymmetric function of its variables, depending on which character of $S_{N}$ the map $\varsigma$ determines (the identity or the sign of the permutation) [20].

This observation suggests the following mathematical setup to more directly account for the indeterminancy in $S_{v}$. Rather than considering the tangent bundle $T \mathcal{M}$, one may consider the collection of Lagrangian submanifolds over the densities, which may be given a symplectic structure. A Lagrangian submanifold $L \subseteq T^{*} \mathcal{M}$ which is the graph of the closed form $\omega$ is then said to be quantizable in case

$$
\int_{\gamma} \omega=2 \pi k(\gamma), \quad k(\gamma) \in \mathbf{Z}
$$

for each closed loop $\gamma$ in $M$. In other words, the de Rham cohomology class $[\omega / 2 \pi]$ in $H^{1}(M, \mathbf{R})$ lies in the image of the singular cohomology $H^{1}(M, \mathbf{Z})$. Since the Lagrangian property is preserved by Hamiltonian vector fields, these observations suggest a dynamical approach to obtaining representations of diffeomorphism groups using the machinery of geometric quantization.

7. Acknowledgments. This paper was written under the direction of Professor Edward Nelson. Thanks are due to Professors Jerrold Marsden and John Mather for helpful and interesting conversations. 


\section{BIBLIOGRAPHY}

1. R. Abraham and J. E. Marsden, Foundations of mechanics, 2nd ed., Benjamin Cummings, Reading, Mass., 1978.

2. M. Adams, T. Ratiu, and R. Schmid, The Lie group structure of diffeomorphism groups and invertible Fourier integral operators, with applications, Infinite Dimensional Groups with Applications, Math. Sci. Res. Inst. Publ. Ser., vol. 2, Springer-Verlag, New York, 1985, pp. $1-69$.

3. V. I. Arnol'd, Mathematical methods of classical mechanics, Graduate Texts in Math., vol. 60, Springer-Verlag, New York, 1978.

4. D. Bakry and M. Emery, Inégalités de Sobolev pour un semi-groupe symétrique, C. R. Acad. Sci. Paris 301 (1985), 411-413.

5. E. A. Carlen, Conservative diffusions, Comm. Math. Phys. 94 (1984), 293-315.

6. D. G. Ebin and J. E. Marsden, Groups of diffeomorphisms and the flow of an incompressible fluid, Ann. of Math. (2) 92 (1970), 102-163.

7. R. Green and K. Shiohama, Diffeomorphisms and volume preserving embeddings of non-compact manifolds, Trans. Amer. Math. Soc. 255 (1979), 403-414.

8. F. Guerra and L. Morato, Quantization of dynamical systems and stochastic control theory, Phys. Rev. D (1983), 1774-1786.

9. V. Guillemin and S. Sternberg, Geometric asymptotics, Math. Surveys, vol. 14, Amer. Math. Soc., Providence, R.I., 1977.

10. __ The moment map and collective motion, Ann. Phys. 127 (1980), 220-253.

11. R. S. Hamilton, The inverse function theorem of Nash and Moser, Bull. Amer. Math. Soc. (N.S.) 7 (1982), 65-222.

12. N. Ikeda and S. Watanabe, Stochastic differential equations and diffusion processes, NorthHolland, Amsterdam, 1981.

13. S. Kobayashi and K. Nomizu, Foundations of differential geometry, vol. II, Wiley, New York, 1969.

14. S. Lang, Differential manifolds, Addison-Wesley, Reading, Mass., 1972.

15. J. Marsden, T. Ratiu, and A. Weinstein, Semidirect products and reduction in mechanics, Trans. Amer. Math. Soc. 281 (1984), 147-177.

16. J. Milnor, Morse theory, Ann. of Math. Stud., No. 51, Princeton Univ. Press, Princeton, N.J., 1969.

17. __, Remarks on infinite dimensional Lie groups, Relativité, Groupes et Topologie (B. S. DeWitt and R. Stora, eds.), North-Holland, Amsterdam, 1984.

18. J. Moser, On the volume elements of a manifold, Trans. Amer. Math. Soc. 120 (1965), 286-294.

19. E. Nelson, Tensor analysis, Princeton Univ. Press, Princeton, N.J., 1969.

20. __ Quantum fluctuations, Princeton Univ. Press, Princeton, N.J., 1985.

21. _ Field theory and the future of stochastic mechanics, Internat. Conf. Stochastic Processes in Classical and Quantum Systems, Ascona, June 24-29, 1985.

22. A. Weinstein, Lectures on symplectic manifolds, CBMS Regional Conf. Ser. in Math., no. 29, Amer. Math. Soc., Providence, R.I., 1977.

23. __ The local structure of Poisson manifolds, J. Differential Geom. 18 (1983), 523-557.

\footnotetext{
Department of Mathematics, HaRVard University, Cambridge, MassaChuSETTS 02138
} 